\title{
Multi-centered invariants, plethysm and grassmannians
}

\author{
Sergio L. Cacciatori ${ }^{a, b}$ Alessio Marrani $^{c}$ and Bert van Geemen ${ }^{d}$ \\ ${ }^{a}$ Dipartimento di Scienze ed Alta Tecnologia, Università degli Studi dell'Insubria, \\ Via Valleggio 11, I-22100 Como, Italy \\ ${ }^{b}$ INFN, Sezione di Milano, \\ Via Celoria 16, 20133 Milano, Italy \\ ${ }^{c}$ Physics Department, Theory Unit, CERN, \\ CH 1211, Geneva 23, Switzerland \\ ${ }^{d}$ Dipartimento di Matematica, Università di Milano, \\ Via Saldini 50, I-20133 Milano, Italy \\ E-mail: sergio.cacciatori@uninsubria.it, alessio.marrani@cern.ch, \\ lambertus . vangeemen@unimi.it
}

ABSTRACT: Motivated by multi-centered black hole solutions of Maxwell-Einstein theories of (super)gravity in $D=4$ space-time dimensions, we develop some general methods, that can be used to determine all homogeneous invariant polynomials on the irreducible $\left(S L_{h}(p, \mathbb{R}) \otimes G_{4}\right)$-representation $(\mathbf{p}, R)$, where $p$ denotes the number of centers, and $S L_{h}(p, \mathbb{R})$ is the "horizontal" symmetry of the system, acting upon the indices labelling the centers. The black hole electric and magnetic charges sit in the symplectic representation $R$ of the generalized electric-magnetic ( $U$-)duality group $G_{4}$.

We start with an algebraic approach based on classical invariant theory, using Schur polynomials and the Cauchy formula. Then, we perform a geometric analysis, involving Grassmannians, Plücker coordinates, and exploiting Bott's Theorem.

We focus on non-degenerate groups $G_{4}$ "of type $E_{7}$ " relevant for (super)gravities whose (vector multiplets') scalar manifold is a symmetric space. In the triality-symmetric stu model of $\mathcal{N}=2$ supergravity, we explicitly construct a basis for the 10 linearly independent degree-12 invariant polynomials of 3-centered black holes.

KEywords: Black Holes, Supergravity Models, Black Holes in String Theory, Global Symmetries

ARXIV EPRINT: 1211.3432 


\section{Contents}

1 Introduction 1

2 Algebraic approach 5

2.1 Invariant theory 5

2.1.1 The Schur polynomials 5

2.1.2 Traces of $G L$-representations $\quad 6$

2.1.3 Decomposing $S^{k}(U \otimes V) \quad 7$

2.2 Application to $p$-centered black holes 8

2.2.1 Homogeneity $k=2 \quad 9$

2.2.2 Homogeneity $k=3$ for $G_{4}=E_{7} \quad 9$

$\begin{array}{lll}2.3 & \text { Examples } & 11\end{array}$

2.3.1 $G_{4}=E_{7}, R=\mathbf{5 6} \quad 12$

2.3.2 $G_{4}=\operatorname{Sp}(6, \mathbb{R}), R=\mathbf{1 4}^{\prime} \quad 14$

$\begin{array}{ll}2.3 .3 G_{4}=\mathrm{SO}(12), R=\mathbf{3 2}^{(\prime)} & 15\end{array}$

2.3.4 $G_{4}=\mathrm{SU}(6), R=\mathbf{2 0} \quad 16$

2.3.5 $G_{4}=\mathrm{SL}(2, \mathbb{R}) \times \mathrm{SL}(2, \mathbb{R}) \times \mathrm{SL}(2, \mathbb{R}), R=(\mathbf{2}, \mathbf{2}, \mathbf{2}) \quad 17$

3 Geometric interpretation $\quad 19$

$\begin{array}{ll}3.1 \text { Grassmannians } & 19\end{array}$

3.1.1 Invariants of $S L_{h}(p) \times G_{4}$ in $\left(\mathbb{R}^{p}\right) \otimes R \quad 19$

3.1.2 From tensors to planes 20

$\begin{array}{ll}3.1 .3 & \text { The Plücker map } \\ \end{array}$

4 3-centered $s t u$ black holes

4.1 Invariant from Cayley's hyperdeterminant: $S^{4}\left(S_{1^{3}}((\mathbf{2}, \mathbf{2}, \mathbf{2}))\right)$

4.2 Other invariants from $S^{2}\left(S_{2^{3}}((\mathbf{2}, \mathbf{2}, \mathbf{2}))\right) \quad 25$

$\begin{array}{lll}4.3 & \text { Explicit construction } & 26\end{array}$

4.3.1 The representation $V\left(a_{1}, a_{2}, a_{3}\right) \quad 27$

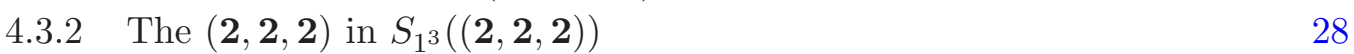

4.3.3 The $(\mathbf{1}, \mathbf{1}, \mathbf{3})^{\oplus 3}$ in $S_{2^{3}}((\mathbf{2}, \mathbf{2}, \mathbf{2})) \quad 28$

$\begin{array}{lll}\text { 4.3.4 stu Triality } & 29\end{array}$

\section{Introduction}

The Attractor Mechanism [1-5], originally discovered in $\mathcal{N}=2, D=4$ Maxwell-Einstein supergravity and then investigated in other extended supergravities as well as in nonsupersymmetric theories of gravity (see e.g. [6-9] for reviews and list of refs.), plays a central role in the physics of extremal black holes (BHs), as well as of (intersecting configurations 
of) extremal black $p$-branes [10], also in $D>4$ space-time dimensions. In its simplest framework, namely in presence of Abelian vectors and scalar fields in the background of an extremal $\mathrm{BH}$, the area of the event horizon can be expressed purely in terms of the fluxes of the 2-form Abelian field strengths and of their duals, whose fluxes define the magnetic and electric $\mathrm{BH}$ charges, fitting a symplectic vector $Q$. The dynamics of the scalar fields exhibits an attractor phenomenon, namely the value of the field at the $\mathrm{BH}$ event horizon is completely determined in terms of the magnetic and electric charges, regardless of the initial (boundary) conditions defined for the flow at spatial infinity. ${ }^{1}$ In general, the near-horizon attractor dynamics can be reformulated in terms of critical points of a BH effective potential [5], which in presence of an underlying local supersymmetry also enjoys a geometric interpretation in terms of central charge(s) and matter charges (if any).

The vector space of electric-magnetic $\mathrm{BH}$ charges generally defines an irreducible ${ }^{2}$ representation (irrep.) space $R$ for the generalized electric-magnetic $(U-)$ duality ${ }^{3}$ group $G_{4}$. Under the action of $G_{4}$, the irrep. space $R$ undergoes a stratification into orbits, which in turn are in correspondence with classes of BHs, with both regular and vanishing near-horizon geometry (corresponding to "large" and "small" BHs, respectively); thus, the classification of $G_{4}$-orbits in $R$ results in a group-theoretical characterization of BH solutions themselves. In Maxwell-Einstein supergravity theories whose scalar manifold is a symmetric space $G_{4} / H_{4}$ (with $H_{4}$ being the maximal compact subgroup of $G_{4}$ ), the classification of orbits can be algebraically achieved in terms of constraints imposed on the unique [18] algebraically independent $G_{4}$-invariant homogeneous polynomial $\mathcal{I}$ in the irrep. $R$ (see e.g. [19-22], as well as [23] for a recent résumé and a list of refs.).

Within this rather broad class of $D=4$ theories, $\mathcal{I}$ is a quadratic polynomial $\left(\mathcal{I}=\mathcal{I}_{2}\right)$ for $\mathcal{N}=2$ minimally coupled $[24,25]$ as well as for $\mathcal{N}=3$ [26] supergravity. In the remaining $D=4$ theories with symmetric scalar manifolds, $G_{4}$ can be characterized (in terms of $R$ ) as a group "of type $E_{7}$ " [27-33]. In particular, the charge representation $R$ satisfies

$$
\operatorname{dim} \wedge^{2} R=\operatorname{dim} S^{4} R=1
$$

Namely, the flux irrep. $R$ is symplectic (i.e., endowed with a unique symplectic structure $\mathbb{C}_{[M N]}:=\mathbf{1} \in \wedge^{2} R=: R_{a}^{\otimes 2}$, as it generally holds in $\left.D=4\right)$, and it exhibits a unique, algebraically independent, degree- 4 homogeneous invariant polynomial ${ }^{4} \mathcal{I}=\mathcal{I}_{4}$, related to a rank-4 completely symmetric $G_{4}$-invariant tensor (the so-called $K$-tensor [34-37]) $K_{(M N P Q)}:=1 \in S^{4} R=: R_{s}^{\otimes 4}$. Simple and semi-simple non-degenerate $U$-duality groups

\footnotetext{
${ }^{1}$ Some exception/violations of the Attractor Mechanism include e.g. the existence of basins of attraction/area codes [11-13] as well as of moduli spaces/flat directions of attractor flows [10, 14].

${ }^{2}$ This strictly holds for unified theories, in which all Abelian 2-form field strengths (and their duals) transform in an irrep. of $G_{4}$; the following reasoning can be easily generalized to non-unified frameworks.

${ }^{3}$ Here $U$-duality is referred to as the "continuous" symmetries of $[15,16]$. Their discrete versions are the $U$-duality non-perturbative string theory symmetries introduced by Hull and Townsend [17].

${ }^{4}$ Actually, this characterizes $G_{4}$ (which can be simple or semi-simple) as a non-degenerate group "of type $E_{7}$ ". The "degeneration" of the $U$-duality symmetry in some $\mathcal{N}=2$ theories $[24,25]$ and in $\mathcal{N}=$ 3 [26] supergravity, and its relation to the minimal coupling of vector and scalar fields in Maxwell-Einstein (super)gravity theories in $D=4$ has recently been investigated in [32].
} 
$G_{4}$ "of type $E_{7}$ " relevant to the class of $D=4$ Maxwell-Einstein (super)gravity theories under consideration are listed in table 1 at the start of section 2.3.

The properties of the quartic polynomial $\mathcal{I}_{4}$ constructed from the $K$-tensor have been exploited in order to characterize in an algebraic way the various scalar flows in the background of extremal single-centered BHs [19-22]. The classification can be extended to multi-centered BHs [38-47]. In the case of 2-centered solutions, a group theoretical study of the invariant structures which can be defined in the vector space of electric-magnetic fluxes has been started in [48], and then developed in [33, 35-37]; the connection between 2-centered invariant structures for the so-called stu model $[49,50]$ of $\mathcal{N}=2, D=4$ supergravity and Quantum Information Theory has then been investigated in [51]. Furthermore, relations between the $K$-tensor of the stu model (giving rise to the so-called Cayley's hyperdeterminant [54-56]) and elliptic curves has been recently studied in [57], and extended to the 2-centered case in [51].

Besides the importance of the symplectic product $\mathcal{W}$ (see eq. (2.17) below) in order to define mutually non-local charge vectors pertaining to different centers [38, 39], the physical relevance of some higher-order $U$-invariant polynomials has been suggested in recent investigations [46], and further study in such a direction is surely deserved in order to unravel their role e.g. in the spatial structure of general stationary almost-BPS [43, 45, 47] and composite non-BPS [45-47] multi-centered BH flows, with flat $D=3$ spatial slices as well as non-flat ones $[44,58,59]$.

In the case of $\mathrm{BH}$ solutions with $p$ centers, the $U$-duality group $G_{4}$ acts on $p$ copies of $R$; correspondingly, the charge vectors $Q^{a}$ carry an index referring to the relevant center $(a=1, \ldots, p)$, and one has to consider polynomial invariants in the $p \operatorname{dim} R$ coordinates on $R^{p}$. Thus, a "horizontal" symmetry ${ }^{5} S L_{h}(p, \mathbb{R})$, commuting with $G_{4}$, naturally occurs. This was firstly introduced in [48], and it acts on the index labelling the various centers, in such a way that $G_{4}$-invariant polynomials generally decompose into $S L_{h}(p, \mathbb{R})$-irreps. In the 2-centered case $(p=2)$, as mentioned, the problem of determining a complete basis for the ring of $\left(S L_{h}(p, \mathbb{R}) \times G_{4}\right)$-invariant homogeneous polynomials has been solved in [48] and [35], respectively for semi-simple and simple non-degenerate groups "of type $E_{7}$ " occurring as $U$-duality groups in $D=4$ supergravities with symmetric scalar manifolds. ${ }^{6}$ Actually, the same results had been obtained, within a completely different approach based on nilpotent orbits, by Kac many years ago in [18]; therein, it was also shown that the complete basis composed by polynomials whose homogeneity degree is the lowest possible is also finitely generating, namely all other higher-order invariant polynomials are simply polynomials in the elements of the basis.

For example, in the 2-centered simple case $[18,35]$ there are 7 algebraically independent $U$-invariant polynomials, which form a minimal degree complete basis for the corresponding ring; out of them, 5 are homogeneous of degree 4 and they are arranged into a $\mathbf{5}$ (spin $s=2$ ) irrep. of the 2-centered "horizontal" symmetry $S L_{h}(2, \mathbb{R})$, while the remaining ones are polynomials homogeneous of degree 2 and 6 that are $S L_{h}(2, \mathbb{R})$-invariant (the one of

\footnotetext{
${ }^{5}$ The subscript " $h$ " stands for "horizontal" throughout.

${ }^{6}$ The same problem was solved, for a generic number $p$ of centers, in [37, 60] for simple, degenerate groups "of type $E_{7}$ " occurring in $\mathcal{N}=2$ minimally coupled as well as in $\mathcal{N}=3$ supergravity in $D=4$.
} 
degree 2 is nothing but the symplectic product $\mathcal{W}$ defined in (2.17) below). Out of these $7 G_{4}$-invariants, one can construct 4 algebraically independent $\left(S L_{h}(2, \mathbb{R}) \times G_{4}\right)$-invariant polynomials, homogeneous of degree 2, 6, 8 and 12 [18,35]. With some abuse of language, $\left(S L_{h}(p, \mathbb{R}) \times G_{4}\right)$-invariants have been usually named "horizontal" invariants.

In the (2-centered) semi-simple case [18, 37, 48], further lower-order horizontal invariant structures arise as a consequence of the factorization of the $U$-duality symmetry $G_{4}$; a particular, noteworthy example is provided by the aforementioned stu model, exhibiting a triality symmetry $[49,50]$, which should be modded out in order to obtain invariant structures relevant for BHs (cfr. the treatment of [48] vs. [51], as well as the treatment in sections 2.3.5 and 4.3.4).

Although some general properties can be inferred from elementary group theoretical considerations, a systematic study and classification of $(p>2)$-centered solutions in terms of $\left(S L_{h}(p, \mathbb{R}) \times G_{4}\right)$-orbits is still lacking.

The aim of the present paper is to start developing some general methods that can be used to determine all invariants associated to $p$-centered $\mathrm{BH}$ solutions, for a generic $p$. In particular, we will be interested in $p$-centered horizontal invariants, namely homogeneous $\left(S L_{h}(p, \mathbb{R}) \times G_{4}\right)$-invariant polynomials on the irrep. $\mathbb{R}^{p} \otimes R=:(\mathbf{p}, R)$ of the overall symmetry $S L_{h}(p, \mathbb{R}) \times G_{4}$ itself. The invariant polynomials homogeneous of degree $k$ are clearly related to the $\left(S L_{h}(p, \mathbb{R}) \times G_{4}\right)$-invariant tensors in the $k$-th completely symmetric ${ }^{7}$ power $S^{k}\left(\mathbb{R}^{p} \otimes R\right)=:(\mathbf{p}, R)_{s}^{\otimes k}$. This allows for the exploitation of the classical invariant theory (for which we will mainly refer to the book [52]).

Let us finally recall that in general, given a representation $V$ of a group $G$, plethysm is the study of the decompositions into irreducible $G$-representations of $V \otimes V, \wedge{ }^{k} V, \operatorname{Sym}^{k}(V)$ and, more generally, of Schur functors applied to $V$ (cfr. e.g. [53]). Plethystic formulas are thus exactly what we need in our investigation, and this justifies the title of our paper.

The plan of the paper is as follows.

In section 2 we use the representation theory of a product group $\mathcal{G} \times G$ in order to determine the corresponding invariant structures. We first recall some general facts about invariant theory and, in particular, the characterization of the $(\mathcal{G} \times G)$-invariants in the symmetric products $S^{k}(U \otimes V)$ of the irreps. $U$ and $V$ of $\mathcal{G}$ and $G$, respectively. By applying these methods to the case $\mathcal{G}=S L_{h}(p, \mathbb{R})$ and $G=G_{4}$ relevant to $p$-centered $(\mathrm{BH})$ solutions in $D=4$ supergravity, we can then count $\left(S L_{h}(p, \mathbb{R}) \times G_{4}\right)$-invariants ${ }^{8}$ for all relevant generic, simple cases.

Next, in section 3, we present a geometric analysis of the invariants. We show that in the $p$-centered case the invariants can be determined by using the $\operatorname{Grassmannian} \operatorname{Gr}(p, R)$ of $p$-planes in $R$. This Grassmannian is embedded in a projective space by its Plücker coordinates, which are global sections of a line bundle $L$ on $\operatorname{Gr}(p, R)$. For any positive

\footnotetext{
${ }^{7}$ The subscript " $s$ " (" $a$ ") stands for symmetric (antisymmetric) throughout.

${ }^{8} \mathrm{Up}$ to a certain order, fixed by the available computing power (see analysis in section 2.3 ).
} 
integer $a$, the group $G L(R)$, and thus ${ }^{9}$

$$
G_{4} \subset S p(R) \subset S L(R) \subset G L(R),
$$

acts on the sections $\Gamma\left(\operatorname{Gr}(p, R), L^{\otimes a}\right)$. These sections are homogeneous polynomials of degree $a$ in the Plücker coordinates. Our geometric characterization of the $\left(S L_{h}(p, \mathbb{R}) \times G_{4}\right)$ invariant polynomials, in combination with Bott's theorem [53], shows that all these invariants are given by $\left(S L_{h}(p, \mathbb{R}) \times G_{4}\right)$-invariant sections. In particular, the $\left(S L_{h}(p, \mathbb{R}) \times G_{4}\right)$ invariant polynomials are generated by homogeneous polynomials in the Plücker coordinates.

Finally, in section 4, we present an application of the methods developed in sections 2 and 3: in the semi-simple, triality-symmetric $\mathcal{N}=2, D=4$ stu model, we compute a basis for the 10-dimensional vector space of $\left(S L_{h}(3, \mathbb{R}) \times \mathrm{SL}(2, \mathbb{R})^{3}\right)$-invariant polynomials homogeneous of degree 12 for 3-centered BHs; the physical issue of invariance under the symmetric group $S_{3}$, implementing the triality symmetry acting on the three copies of $\operatorname{SL}(2, \mathbb{R})$ in the $U$-duality group $G_{4}=\operatorname{SL}(2, \mathbb{R})^{3}$, is considered in sections 2.3.5 and 4.3.4.

\section{Algebraic approach}

\subsection{Invariant theory}

In order to tackle the problem of determining the invariants associated to multi-centered $\mathrm{BH}$ solutions, we will make use of the classical invariant theory. Let us first collect some basic facts on how to find invariants in $U \otimes V$ for the action of the group $G L(U) \times G L(V)$; as mentioned above, we will mainly refer to the book [52], to which we address the reader for further details and a list of refs.

\subsubsection{The Schur polynomials}

A partition $\lambda$ of an integer $m \in \mathbb{Z}_{>0}$, denoted as $\lambda \vdash m$, is a non-increasing sequence $\lambda=\left(p_{1}, \ldots, p_{N}\right)$ of integers $p_{i} \in \mathbb{Z}_{\geq 0}$ such that $\sum_{i=}^{N} p_{i}=m$. The number of non-zero elements in $\lambda$ is denoted by $h t(\lambda):=n$, so $p_{i}=0$ for $i>n$.

The Schur polynomial $S_{\lambda}$ in $N$ variables $x_{1}, \ldots, x_{N}$, where $N \geq n:=h t(\lambda)$, is the symmetric polynomial, with integral coefficients, defined as the quotient ([52], 2.3.2)

$$
S_{\lambda}(x):=\frac{A_{\lambda+\rho}(x)}{V(x)},
$$

where the partition $\lambda+\rho$ is defined as $\lambda+\rho:=\left(p_{1}+N-1, p_{2}+N-2, \ldots, p_{N}\right)$, and $A_{\lambda+\rho}(x)$ and $V(x)$ (Vandermonde determinant) are two anti-symmetric polynomials in $x_{1}, \ldots, x_{N}$,

\footnotetext{
${ }^{9}$ As also recently discussed in [61], the maximal (but generally non-symmetric) embedding $G_{4} \subset \operatorname{Sp}(R)$ (which in supergravity is named Gaillard-Zumino [62] embedding) can be regarded as a consequence of the following Theorem by Dynkin (Th. 1.5 of [63], more recently discussed e.g. in [64]): every irreducible group of unimodular linear transformations of the $N$-dimensional complex space (namely, a group of transformations which does not leave invariant a proper subspace of such a space) is maximal either in $\operatorname{SL}(N)$ (if the group does not have a bilinear invariant), or in $\operatorname{Sp}(N)$ (if it has a skew-symmetric bilinear invariant), or in $O(N)$ (if it has a symmetric bilinear invariant). Exceptions to this rule are listed in table VII of [64].
} 
respectively given by

$$
A_{\left(m_{1}, \ldots, m_{N}\right)}(x):=\sum_{\sigma \in S_{N}} \epsilon_{\sigma} x_{\sigma(1)}^{m_{1}} x_{\sigma(2)}^{m_{2}} \cdots x_{\sigma(N)}^{m_{N}},
$$

where $S_{N}$ is the group of permutations of $N$ variables and $\epsilon_{\sigma}$ is the permutation parity, and

$$
V(x):=\prod_{1 \leq i<j \leq N}\left(x_{i}-x_{j}\right) .
$$

Note that $S_{\lambda}=0$ if $h t(\lambda)>N$.

As from Th. 1 in [52], 2.3.2, the Schur polynomials $S_{\lambda}$ with $\lambda \vdash m$ and $h t(\lambda) \leq N$ are a basis of the polynomials in $N$ variables which are homogeneous of degree $m$ and are invariant under permutations of the variables $x_{1}, \ldots, x_{N}$. Examples are provided by the elementary symmetric functions

$$
\begin{array}{rlrl}
S_{1^{h}} & =\sum_{1 \leq i_{1}<\ldots<i_{h} \leq N} x_{i_{1}} x_{i_{2}} \cdots x_{i_{h}}, & \lambda=1^{h}:=(\underbrace{1, \ldots, 1}_{h}, 0, \ldots, 0) ; \\
S_{k}=\sum_{1 \leq i_{1} \leq \ldots \leq i_{k} \leq N} x_{i_{1}} x_{i_{2}} \cdots x_{i_{k}}, & \lambda=k:=(\underbrace{k, \ldots, 0}_{N-1}),
\end{array}
$$

which differ only in the possibility to consider or not the same values for at least a pair of indices in the string $i_{1}, \ldots, i_{h}$.

\subsubsection{Traces of $G L$-representations}

Let $V$ be a vector space of dimension $N$ with basis $v_{1}, \ldots, v_{N}$, and let $y:=\left(y_{1}, \ldots, y_{N}\right) \in$ $\left(\mathbb{C}^{*}\right)^{N}$ act by $\operatorname{diag}\left(y_{1}, \ldots, y_{N}\right)$ on $V$.

A partition $\lambda$ with $h t(\lambda) \leq N$ defines an irreducible representation $S_{\lambda}(V)$ of $G L(V)$ which is a summand of $\otimes^{m} V$ where $\lambda \vdash m$ ([52] 9.3.1, (3.1.3)). If $h t(\lambda)>N$, then $S_{\lambda}(V)=0$. Moreover, any irreducible representation of $G L(V)$ is isomorphic to an $S_{\lambda}(V)$ for a unique partition $\lambda$ with $h t(\lambda) \leq N([52], 9.8 .1)$. The trace of $\operatorname{diag}\left(y_{1}, \ldots, y_{N}\right) \in\left(\mathbb{C}^{*}\right)^{N}$ (i.e. the standard maximal torus of $G L(V))$ on the irreducible representation $S_{\lambda}(V)$ is the Schur polynomial $S_{\lambda}\left(y_{1}, \ldots, y_{N}\right)$. The dimension of the representation associated to the partition $\left(p_{1}, \ldots, p_{N}\right)$ is $([52], 9.6 .2)$ :

$$
\operatorname{dim} S_{\lambda}(V)=\prod_{1 \leq i<j \leq N} \frac{p_{i}-p_{j}+j-i}{j-i}, \quad \lambda=\left(p_{1}, \ldots, p_{N}\right) .
$$

For instance, $\lambda:=1^{h}$ defines $S_{\lambda}(V):=\wedge^{h} V(2.4)$, the rank- $h$ completely antisymmetric tensor representation of $G L(V)$, which has dimension $\left(\begin{array}{c}N \\ h\end{array}\right)$; in particular, the partition $\lambda=1^{N}$ selects the one-dimensional determinant representation on $\wedge^{N} V$ (realized by the Ricci-Levi-Civita symbol $\epsilon_{i_{1} \ldots i_{N}}$ ).

Another example is provided by the partition $\lambda:=k:=(k, \underbrace{0, \ldots, 0}_{N-1})$, which defines $S_{\lambda}(V):=$ $S^{k} V(2.5)$, the $k$-th symmetric product of $V$, namely the rank- $k$ completely symmetric tensor representation of $G L(V)$. A basis of $S^{k} V$ is provided by $v_{1}^{a_{1}} \cdots v_{N}^{a_{N}}$ with $a_{i} \geq 0$ 
and $\sum_{i=1}^{N} a_{i}=k$, and the action of $y$ on this basis elements is the multiplication by $y_{1}^{a_{1}} \cdots y_{N}^{a_{N}}$. Hence, the trace of $y$ on $S^{k} V$ is the sum of all monomials in $y_{1}, \ldots, y_{N}$ which are homogeneous of degree $k$. As mentioned, this is the Schur polynomial $S_{k}(2.5)$, so $\operatorname{tr}\left(y \mid S^{k} V\right)=S_{k}(y)$. A generating function for these $S_{k}$ can be obtained by noting that

$$
\begin{aligned}
& \left(1+\ldots+y^{a_{1}} t^{a_{1}}+\ldots\right)\left(1+\ldots+y^{a_{2}} t^{a_{2}}+\ldots\right) \ldots\left(1+\ldots+y^{a_{N}} t^{a_{N}}+\ldots\right) \\
= & 1+S_{1}(y) t+S_{2}(y) t^{2}+\ldots+S_{k}(y) t^{k}+\ldots,
\end{aligned}
$$

and it is given by the Molien formula $\left(S_{0}(y)=1 ;[52], 9.4 .3,(4.4 .3)\right)$ :

$$
\prod_{j=1}^{N} \frac{1}{1-y_{j} t}=\sum_{k=0}^{\infty} S_{k}(y) t^{k}
$$

\subsubsection{Decomposing $S^{k}(U \otimes V)$}

A generalization of the Molien formula (2.8), which yields the decomposition of $S^{k}(U \otimes V)$ under $G L(U) \times G L(V)$, is provided by the following formula, due to Cauchy. Let $^{10} m \leq n$ be two positive integers, then:

$$
\prod_{i=1}^{m} \prod_{j=1}^{n} \frac{1}{1-x_{i} y_{j}}=\sum_{\lambda: h t(\lambda) \leq m \leq n} S_{\lambda}\left(x_{1}, \ldots, x_{m}\right) S_{\lambda}\left(y_{1}, \ldots, y_{n}\right) .
$$

The interpretation of the Cauchy formula (2.9) in terms of characters of representations is given e.g. in [52], 9.6.3. Let $U$ and $V$ be vector spaces of dimension $m$ and $n$ respectively, and assume that $m \leq n$. Let $u_{1}, \ldots, u_{m}, v_{1}, \ldots, v_{n}$ be bases of $U, V$ respectively, and let $x \in\left(\mathbb{C}^{*}\right)^{m}, y \in\left(\mathbb{C}^{*}\right)^{n}$ act on these spaces by $\operatorname{diag}\left(x_{1}, \ldots, x_{m}\right), \operatorname{diag}\left(y_{1}, \ldots, y_{n}\right)$. The eigenvalues of $(x, y)$ on $U \otimes V$ are then the $x_{i} y_{j}$ with $1 \leq i \leq m$ and $1 \leq j \leq n$. Thus, Cauchy formula (2.9) implies that

$$
\sum_{k=0}^{\infty} \operatorname{tr}\left((x, y) \mid S^{k}(U \otimes V)\right) t^{k}=\sum_{\lambda: h t(\lambda) \leq m \leq n} S_{\lambda}(x) S_{\lambda}(y) t^{d_{\lambda}}, \quad \lambda \vdash d_{\lambda} .
$$

Using the bijection between traces of irreducible representations and irreducible characters, it follows that there is an isomorphism of $(G L(U) \times G L(V))$-representations:

$$
S^{k}(U \otimes V) \cong \bigoplus_{\lambda \vdash k, h t(\lambda) \leq m} S_{\lambda}(U) \otimes S_{\lambda}(V) .
$$

A particular consequence of the isomorphism (2.11) is that if $\mathcal{G} \times G$ is a subgroup of $G L(U) \times G L(V)$, then the vector space $\left(S^{k}(U \otimes V)\right)^{\mathcal{G} \times G}$ of $(\mathcal{G} \times G)$-invariants in $S^{k}(U \otimes V)$ enjoys the following decomposition:

$$
\left(S^{k}(U \otimes V)\right)^{\mathcal{G} \times G} \cong \bigoplus_{\lambda \vdash k, h t(\lambda) \leq m}\left(S_{\lambda}(U)^{\mathcal{G}}\right) \otimes\left(S_{\lambda}(V)^{G}\right)
$$

\footnotetext{
${ }^{10}$ The formula (2.9) is proven in [52], 2.3.4 for $n=m$, but setting $x_{i}=0$ for $m \leq i \leq n$, the proof holds for $m \leq n$.
} 
since the action of $\mathcal{G} \times G$ on $S_{\lambda}(U) \otimes S_{\lambda}(V)$ preserves the factors. Thus, in order to compute the $(\mathcal{G} \times G)$-invariants, one can compute the $\mathcal{G}$-invariants on all $S_{\lambda}(U)$ and the $G$-invariants on all $S_{\lambda}(V)$, and then combine the results.

Given a partition $\lambda=\left(p_{1}, \ldots, p_{N}\right)$, we define an integer $k \in \mathbb{Z}_{\geq 0}$ and a partition $\mu$ with $h t(\mu) \leq N-1$ by

$$
\lambda=(k, \ldots, k)+\left(k_{1}, \ldots, k_{N-1}, 0\right):=\left(k^{N}\right)+\mu .
$$

Then, the restriction of $S_{\lambda}(V)$ to $\operatorname{SL}(V)$ is isomorphic to $S_{\mu}(V)$, since $\left(k^{N}\right)$ is the $k$-th tensor product of the determinant representation. Ça va sans dire, if $h t(\lambda)>n$, then the definition of $S_{\lambda}(V)$ shows that it is the 0-dimensional vector space.

\subsection{Application to $p$-centered black holes}

As in section 1, let $G_{4}$ be the $U$-duality group acting on the representation $R$ in which the (fluxes of the) Abelian 2-form field strengths and their duals sit, in the background of a $p$-centered black hole solution in the corresponding $D=4$ Maxwell-Einstein (super)gravity theory. Since the "horizontal" [48] group $S L_{h}(p) \equiv S L_{h}(p, \mathbb{R})$ acts on the labels of the centers, in order to determine the invariants associated to the $p$-centered $\mathrm{BH}$ one has to compute the invariants of $\mathcal{G} \times G=S L_{h}(p) \times G_{4}$ on $U \otimes V=\mathbb{R}^{p} \otimes R=:(\mathbf{p}, R)$.

The representation $S_{\lambda}(U)$, where $U=\mathbb{R}^{p}$, of $\mathcal{G}=S L_{h}(p)$, is irreducible (if non-zero), and there are very few cases in which it is the trivial 1-dimensional representation. In fact, recall that $S_{\lambda}(V)=0$ if $h t(\lambda)>p$, whereas if $h t(\lambda)<p$ then $S_{\lambda}(V)$ is an irreducible representation of $G L(V)$, and hence also of $\operatorname{SL}(V)$. Thus $S_{\lambda}(V)^{\mathcal{G}}=0$, unless $S_{\lambda}(V)$ is a power of the 1-dimensional determinant representation of $G L(V)$; namely, unless the partition reads $\lambda=(a, \ldots, a)=:\left(a^{p}\right)$, in which case one has

$$
U=\mathbb{R}^{p} \text { of } \mathcal{G}=S L_{h}(p): \operatorname{dim}\left(S_{\lambda}(U)^{S L_{h}(U)}\right)=1 \quad \Longleftrightarrow \quad \lambda=\left(a^{p}\right)=\underbrace{(a, \ldots, a)}_{p}
$$

for some $a \in \mathbb{Z}_{\geq 0}$, and $\operatorname{dim}\left(S_{\lambda}(U)^{S L_{h}(U)}\right)=0$ otherwise.

In virtue of formula (2.12), this implies that the invariants of $S L_{h}(p) \times G_{4}$ in $S^{k}\left(\mathbb{R}^{p} \otimes R\right)$ must come from the invariants of $G_{4}$ in $S_{\lambda}(R)$ where $\lambda=\left(a^{p}\right)$. As $\left(a^{p}\right) \vdash p a$, it thus also follows $^{11}$

$$
\operatorname{dim}\left(S^{p a}\left(\mathbb{R}^{p} \otimes R\right)^{S L_{h}(p) \times G_{4}}\right)=\operatorname{dim}\left(S_{\left(a^{p}\right)}(R)^{G_{4}}\right),
$$

and there are no invariants ${ }^{12}$ in $S^{k}\left(\mathbb{R}^{p} \otimes R\right)$ if $k$ is not a multiple of $p$. So, if one has a degree- $k\left(S L_{h}(p) \times G_{4}\right)$-invariant homogeneous polynomial in the representation $\mathbb{R}^{p} \otimes R$, then $k$ is a multiple of $p$ (the converse surely does not hold; see e.g. tables (2.31), (2.38), (2.40), (2.42) and (2.45) below). Before explicitly analyzing some cases relevant to supergravity, let us consider the lowest degrees of homogeneity: $k=2$ and $k=3$.

\footnotetext{
${ }^{11}$ In section 3.1 we will discuss in some detail the $G_{4}$-representation $S_{\left(a^{p}\right)}(R)$ which gives rise to all invariants.

${ }^{12}$ Besides the above reasoning, another proof of this fact is the following one : the group $\mathrm{SL}(p, \mathbb{C})$ contains the matrices $\lambda I$ where $\lambda=e^{2 \pi i / p}$, and the element $(\lambda I, I) \in \mathrm{SL}(p) \times G_{4}$ acts as multiplication by the scalar $\lambda$ on $\mathbb{C}^{p} \otimes R$, and hence by $\lambda^{k}$ on $S^{k}\left(\mathbb{C}^{p} \otimes R\right)$.
} 


\subsubsection{Homogeneity $k=2$}

In the case $k=2$, the partitions $\lambda$ with $\lambda \vdash 2$ are $\lambda=(2,0)=: 2$ and $\lambda=(1,1)=: 1^{2}$. Since $S_{2}(V)=S^{2} V$ and $S_{1^{2}}=\wedge^{2} V$, one obtains (provided $\left.h t(\lambda) \leq 2 \leq \min (\operatorname{dim} U, \operatorname{dim} V)\right)$ :

$$
S^{2}(U \otimes V) \cong\left(S^{2} U\right) \otimes\left(S^{2} V\right) \oplus\left(\wedge^{2} U\right) \otimes\left(\wedge^{2} V\right) .
$$

A particular case, in which the term $\left(S^{2} U\right) \otimes\left(S^{2} V\right)$ does not yield any invariant, is provided by 2-centered $(p=2)$ BHs in the framework under consideration, namely for $p=2: U \otimes V=\mathbb{R}^{2} \otimes R$ of $\mathcal{G} \times G=S L_{h}(2, \mathbb{R}) \times G_{4}$. As both $S L_{h}(2, \mathbb{R})$ and $G_{4}$ have an invariant in $\wedge^{2} \mathbb{R}^{2} \cong \mathbb{R}$ and in $\wedge^{2} R$, respectively (namely, both the fundamental spin $s=1 / 2$ irrep. 2 of $S L_{h}(2, \mathbb{R})$ and the irrep. $R$ of $G_{4}$ are symplectic) one obtains one invariant from the term $\left(\wedge^{2} U\right) \otimes\left(\wedge^{2} V\right)$ of (2.16), given by the symplectic product $\mathcal{W}$ in $R$ of $G_{4}$, namely $[35,37,48](a, b=1,2, M, N=1, \ldots, \operatorname{dim} R)$ :

$$
\mathcal{W}:=\left(Q_{1}, Q_{2}\right):=\mathbb{C}_{M N} Q_{1}^{M} Q_{2}^{N}=\frac{1}{2} \epsilon^{a b} \mathbb{C}_{M N} Q_{a}^{M} Q_{b}^{N},
$$

where $\epsilon^{a b}$ is the Ricci-Levi-Civita symbol of $S L_{h}(2, \mathbb{R})$. When $\mathcal{W} \neq 0$, the charge vectors $Q_{1}$ and $Q_{2}$ (respectively pertaining to BH centers 1 and 2) are mutually non-local, and the distance between the two centers in the BPS 2-centered system is fixed [38, 39]. No other algebraically independent invariant polynomial homogeneous of degree $k=2$ arise, since the representations $U=\mathbb{R}^{2}=: \mathbf{2}$ of $S L_{h}(2, \mathbb{R})$ and $V=R$ of $G_{4}$ are irreducible, and thus there are no other invariants in $S^{2} U$ and in $S^{2} V$.

As discussed at the end of section 3 of [35], some $S L_{h}(p, \mathbb{R})$-covariant structures for $p \geqslant$ 3 can be directly inferred from the 2-centered ones. Indeed, the 2-centered representation of spin $s=J / 2$ of $S L_{h}(2, \mathbb{R})$ is in general replaced by the completely symmetric rank- $J$ tensor representation ${ }^{13} S^{J} \mathbf{p}$ of $S L_{h}(p, \mathbb{R})$. On the other hand, for $p$ centers $\mathcal{W}(2.17)$ generally sits in the $\left(\wedge^{2} \mathbf{p}, \mathbf{1}\right)$ of $S L_{h}(p, \mathbb{R}) \times G_{4}$, where $\wedge^{2} \mathbf{p}$ is the rank-2 antisymmetric tensor representation ${ }^{14}$ (which, in the case $p=2$, becomes a singlet).

\subsubsection{Homogeneity $k=3$ for $G_{4}=E_{7}$}

In the case $k=3$, the partitions $\lambda$ with $\lambda \vdash 3$ are $\lambda=(3,0,0)=: 3, \lambda=(2,1,0)=:(2,1)$ and $\lambda=(1,1,1)=: 1^{3}$. Since $S_{3}(V)=S^{3} V$ and $S_{1^{3}}=\wedge^{3} V$, the $G L(V)$-representation $S_{(2,1)}(V)$ is obtained by the decomposition ${ }^{15}$ (cfr. e.g. [52], 9.3.1)

$$
V^{\otimes 3}:=V \otimes V \otimes V \cong\left(S^{3} V\right) \oplus\left(S_{(2,1)}(V)\right)^{\oplus 2} \oplus \wedge^{3} V .
$$

The simplest example is provided once again by $V=\mathbb{R}^{2}=: \mathbf{2}$ of $S L_{h}(2, \mathbb{R})$, for which it holds

$$
\mathbf{2} \otimes \mathbf{2} \otimes \mathbf{2} \cong(\mathbf{3} \oplus \mathbf{1}) \otimes \mathbf{2} \cong(\mathbf{4} \oplus \mathbf{2}) \oplus \mathbf{2},
$$

\footnotetext{
${ }^{13}$ In the case of $G L(p, \mathbb{R})$, this is given by $S_{\lambda}(V)(2.5)$ with $V=\mathbb{R}^{p}=: \mathbf{p}$ and $\lambda:=J:=(J, \underbrace{0, \ldots, 0}_{p-1})$; see below (2.6). The same holds for $\operatorname{SL}(p, \mathbb{R})$.

${ }^{14}$ In the case of $G L(p, \mathbb{R})$, this is given by $S_{\lambda}(V)(2.4)$ with $V=\mathbb{R}^{p}=$ : $\mathbf{p}$ and $\lambda:=1^{2}$; see below (2.6). The same holds for $\mathrm{SL}(p, \mathbb{R})$.

${ }^{15}$ This is generalized to $V^{\otimes n}$ (for a generic $n$ ) e.g. in [52], 9.3.1.
} 
where $\mathbf{4}=: S^{3} V$ is the spin $s=3 / 2$ of $S L_{h}(2, \mathbb{R})$ itself, consistent with the Clebsch-Gordan formula for this group.

Another example, in which we also exploit the physicists' notation of representations by means of their dimension, is provided by $V=V\left(\lambda_{7}\right)=: 56$ (fundamental) irrep. of $G_{4}=E_{7}$. In this case, the following decomposition holds: ${ }^{16}$

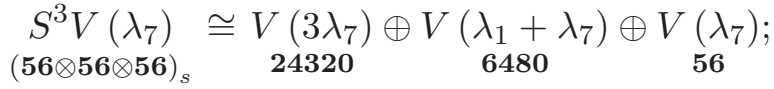

$$
\begin{aligned}
& \underset{(\mathbf{5 6} \otimes \mathbf{5 6} \otimes \mathbf{5 6})_{a}}{\wedge^{3} V\left(\lambda_{7}\right)} \cong \underset{\mathbf{2 7 6 6 4}}{V\left(\lambda_{5}\right)} \oplus \underset{\mathbf{5 6}}{V\left(\lambda_{7}\right) .}
\end{aligned}
$$

On the other hand:

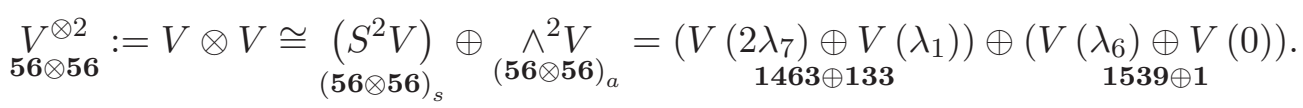

Thus, tensoring once more with $V\left(\lambda_{7}\right)$, one obtains

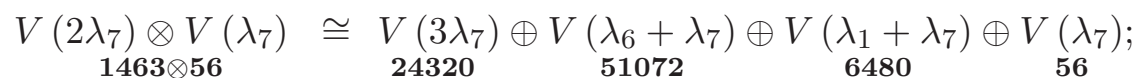

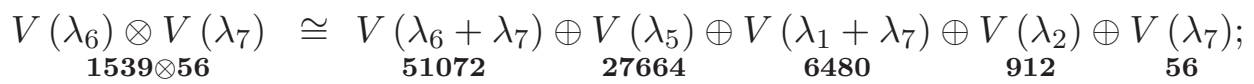

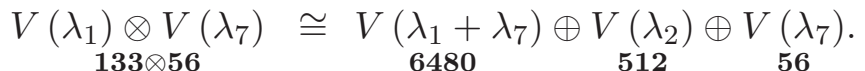

Thus, (2.18) and (2.20)-(2.25) yield

$$
S_{(2,1)}\left(V\left(\lambda_{7}\right)\right) \cong \underset{\mathbf{5 1 0 7 2}}{V\left(\lambda_{6}+\lambda_{7}\right)} \underset{\mathbf{6 4 8 0}}{V\left(\lambda_{1}+\lambda_{7}\right)} \underset{\mathbf{9 1 2}}{V\left(\lambda_{2}\right)} \underset{\mathbf{5 6}}{V\left(\lambda_{7}\right)}
$$

Therefore, we obtained that

$$
\underset{\mathbf{1}}{V(0)} \notin\left\{\begin{array}{l}
S^{3} V\left(\lambda_{7}\right) ; \\
S_{(2,1)}\left(V\left(\lambda_{7}\right)\right) ; \\
\wedge^{3} V\left(\lambda_{7}\right),
\end{array}\right.
$$

and thus there are no $E_{7}$-invariants in $S_{\lambda}\left(V\left(\lambda_{7}\right)\right)$ if $\lambda \vdash 3$. More in general, there are no $E_{7}$-invariants on $V\left(\lambda_{7}\right)^{\otimes n}$ for $n$ odd. Since $S_{\lambda}(V) \subset V^{\otimes n}$ when $\lambda \vdash n$, it follows that there are no $E_{7}$-invariants in $S_{\lambda}\left(V\left(\lambda_{7}\right)\right)$ when $\lambda$ is a partition of an odd (positive) integer $n$.

In other words, there are no invariant polynomials in the fundamental representation $V\left(\lambda_{7}\right)=: \mathbf{5 6}$ of $E_{7}$ with an odd homogeneity degree, as also confirmed by the treatment of section 2.3.1; more in general, this will hold at least for all the (simple and semi-simple) groups "of type $E_{7}$ " which we will consider: there are no invariant polynomials in the relevant irrep. $R$ of $G_{4}$ with an odd homogeneity degree. ${ }^{17}$

\footnotetext{
${ }^{16}$ The weights/roots standard notation of irreps. is used throughout.

${ }^{17}$ The reason can be traced back to the fact that $-I$ on $R$ belongs to $G_{4}$. For instance, it can be checked that the $-I$ in the $\mathbf{5 6}$ of $E_{7}$ preserves the symplectic metric $\mathbb{C}_{[M N]}$ in $\mathbf{5 6}_{a}^{\otimes 2}$ and the quartic symmetric tensor $K_{(M N P Q)}$ in $\mathbf{5 6}_{s}^{\otimes 4}(M, N, P, Q=1, \ldots, 56)$.
} 


\begin{tabular}{|c||c|c|c|}
\hline$J_{3}$ & $G_{4}$ & $R$ & $\mathcal{N}$ \\
\hline \hline$J_{3}^{\mathbb{Q}}, J_{3}^{\mathbb{O}_{s}}$ & $E_{7(-25)}, E_{7(7)}$ & $\mathbf{5 6}$ & 2,8 \\
\hline$J_{3}^{\mathbb{H}}, J_{3}^{\mathbb{H}_{s}}$ & $S O^{*}(12), \mathrm{SO}(6,6)$ & $\mathbf{3 2}^{(\prime)}$ & 2 or 6,0 \\
\hline$J_{3}^{\mathbb{C}}, J_{3}^{\mathbb{C}_{s}}, M_{1,2}(\mathbb{O})$ & $S U(3,3), \mathrm{SL}(6, \mathbb{R}), \mathrm{SU}(1,5)$ & $\mathbf{2 0}$ & $2,0,5$ \\
\hline$J_{3}^{\mathbb{R}}$ & $S p(6, \mathbb{R})$ & $\mathbf{1 4}^{\prime}$ & 2 \\
\hline $\mathbb{R}$ & $S L(2, \mathbb{R})$ & $\mathbf{4}$ & 2 \\
$\left(t^{3}\right.$ model $)$ & & & $2(m$ or $n=2)$ \\
$R \oplus \boldsymbol{\Gamma}_{m-1, n-1}$ & $S L(2, \mathbb{R}) \times \mathrm{SO}(m, n)$ & $(\mathbf{2}, \mathbf{m}+\mathbf{n})$ & $\begin{array}{c}(m \text { or } n=6) \\
0 \text { otherwise }\end{array}$ \\
\hline
\end{tabular}

Table 1. Simple and semi-simple, non-degenerate $U$-duality groups $G_{4}$ "of type $E_{7}$ " [27]. The relevant symplectic irrep. $R$ of $G_{4}$ is also reported. Note that the $G_{4}$ related to split composition algebras $\mathbb{O}_{s}, \mathbb{H}_{s}, \mathbb{C}_{s}$ is the maximally non-compact (split) real form of the corresponding compact Lie group. The corresponding scalar manifolds are the symmetric spaces $\frac{G_{4}}{H_{4}}$, where $H_{4}$ is the maximal compact subgroup (with symmetric embedding) of $G_{4}$. The number of supercharges $\mathcal{N}$ of the resulting supergravity theory in $D=4$ is also listed. The $D=5$ uplift of the $t^{3}$ model (based on $J_{3}=\mathbb{R}$ ) is the pure $\mathcal{N}=2, D=5$ supergravity. $J_{3}^{\mathbb{H}}$ is related to both 8 and 24 supersymmetries, because the corresponding supergravity theories share the very same bosonic sector [25, 65-69].

\section{$2.3 \quad$ Examples}

generalized electric-magnetic $\left(U\right.$-)duality group $G_{4}$; as done above, we denote the relevant $G_{4}$-representation in which the (fluxes of the) Abelian 2-form field strengths (and their duals) sit by ${ }^{18} V=R$, and we will specify it case by case.

In particular, we here consider the class of groups "of type $E_{7}$ " [27] which can be characterized as conformal groups of rank-3, simple Euclidean Jordan algebras $J_{3}^{\mathbb{A}}$ or $J_{3}^{\mathbb{A}_{s}}$, or equivalently as the automorphism group of the Freudenthal triple system (FTS) $\mathfrak{M}\left(J_{3}\right)$

\footnotetext{
${ }^{18}$ It is worth pointing out that the irrep. $R$ is real for the very non-compact real forms of $G_{4}$ pertaining to the relevant $U$-duality groups, while usually for the other (non-compact) real forms it is pseudo-real (quaternionic). This reality property can e.g. be inferred from the corresponding (symmetric) embeddings into $G_{3}$, the relevant $U$-duality symmetry in $D=3$ space-time dimensions.

As an example, let us consider the fundamental representation $R=\mathbf{5 6}$ of $E_{7}$ : it is real for the relevant noncompact real forms $E_{7(7)}$ (split) and $E_{7(-25)}$ (minimally non-compact), while it is pseudo-real (quaternionic) for $E_{7(-133)}$ and $E_{7(-5)}$. Indeed, while $E_{7(7)}$ and $E_{7(-25)}$ respectively embed into $E_{8(8)}$ and $E_{8(-24)}$ through a $\mathrm{SL}(2, \mathbb{R})$ commuting factor:
}

$$
E_{8(8)} \supset E_{7(7)} \times \mathrm{SL}(2, \mathbb{R}), \quad E_{8(-24)} \supset E_{7(-25)} \times \mathrm{SL}(2, \mathbb{R}),
$$

$E_{7(-133)}$ and $E_{7(-5)}$ embed into $E_{8(-24)}$ and $E_{8(8)}$ through an $\mathrm{SU}(2)$ factor:

$$
\begin{aligned}
E_{8(-24)} & \supset E_{7(-133)} \times \mathrm{SU}(2), \quad E_{8(-24)} \supset E_{7(-5)} \times \mathrm{SU}(2) ; \\
E_{8(8)} & \supset E_{7(-5)} \times \mathrm{SU}(2) .
\end{aligned}
$$


constructed over such algebras [70-72]:

$$
G_{4}=\operatorname{Conf}\left(J_{3}\right)=\operatorname{Aut}\left(\mathfrak{M}\left(J_{3}\right)\right) .
$$

$\mathbb{A}$ denotes the division algebras $\mathbb{A}=\mathbb{O}, \mathbb{H}, \mathbb{C}, \mathbb{R}$, while $\mathbb{A}_{s}$ denotes the corresponding split composition algebras $\mathbb{A}_{s}=\mathbb{O}_{s}, \mathbb{H}_{s}, \mathbb{C}_{s}, \mathbb{R}$. The representation $R$ pertains to $\mathfrak{M}\left(J_{3}\right)$, and its dimension is $6 q+8$, where the parameter $q=\operatorname{dim}_{\mathbb{R}} \mathbb{A}_{(s)}=8,4,2,1$ for $\mathbb{A}_{(s)}=$ $\mathbb{O}_{(s)}, \mathbb{H}_{(s)}, \mathbb{C}_{(s)}, \mathbb{R}$, respectively. These class of groups "of type $E_{7}$ " has been recently studied as $U$-duality symmetries in the context of $D=4$ locally supersymmetric theories of gravity in [30-32], as well as gauge (and global) symmetries in particular $D=3$ gauge theories [61].

An exception is provided by the stu model $[49,50]$ (section 2.3.5), whose triality symmetry is exploited within a particular case in section 4 .

From section 2.2, it is here worth recalling that in general there are no polynomial invariants of $(\mathbf{p}, R)$ of $S L_{h}(p, \mathbb{R}) \times G_{4}$ with homogeneity degree $k$ if $k$ is not a multiple of $p$.

\subsection{1 $G_{4}=E_{7}, R=\mathbf{5 6}$}

This is the prototypical case of groups "of type $E_{7}$ " [27]. In supergravity, this is related to the $D=4$ theories with symmetric scalar manifold, based on the FTS $\mathfrak{M}\left(J_{3}^{\mathbb{Q}}\right)$ (exceptional $\mathcal{N}=2$ Maxwell-Einstein theory, with $\left.G_{4}=E_{7(-25)}[65-67]\right)$ and $\mathfrak{M}\left(J_{3}^{\mathbb{Q}_{s}}\right)(\mathcal{N}=8$ maximal supergravity, with $\left.G_{4}=E_{7(7)}[15,16,73]\right)$, where $J_{3}^{\mathbb{Q}}$ and $J_{3}^{\mathbb{Q}_{s}}$ are rank-3 Euclidean Jordan algebras over the octonions $\mathbb{O}$ and split octonions $\mathbb{O}_{s}$, respectively.

The dimension $\operatorname{dim} S_{\left(a^{p}\right)}(R)^{E_{7}}$ for the partition $\lambda=a^{p}$ and $R=V\left(\lambda_{7}\right)=: \mathbf{5 6}$ (fundamental irrep.) can be computed e.g. by using the software LiE, ${ }^{19}$ typing the command ${ }^{20}$

$$
\operatorname{plethysm}([a, \ldots, a],[0,0,0,0,0,0,1], \mathrm{E} 7)[1] \text {. }
$$

The "[1]" at the end corresponds to the lowest representation. The output of the command is an integer, which we denote by $d$, times $X\left[b_{1}, \ldots, b_{7}\right]$, where $X\left[b_{1}, \ldots, b_{7}\right]$ indicates the representation with highest weight $b_{1} \lambda_{1}+\cdots+b_{7} \lambda_{7}$, the $\lambda_{i}$ being the fundamental weights $(i=1, \ldots, 7)$. If all $b_{i}$ 's are zero, then one has found polynomial invariants of homogeneity degree $p a$ in $p \operatorname{dim} R=56 p$ variables; the real dimension of the vector space of such invariants is given by (recall $(2.15)$ )

$$
\begin{aligned}
\operatorname{dim}\left[S_{\lambda=a^{p}}\left(V\left(\lambda_{7}\right)\right)\right]^{E_{7}} & =\operatorname{dim}\left[S^{p a}\left(p, V\left(\lambda_{7}\right)\right)\right]^{S L_{h}(p, \mathbb{R}) \times E_{7}} \\
& =\operatorname{dim}\left[S^{p a}(\mathbf{p}, \mathbf{5 6})\right]^{S L_{h}(p, \mathbb{R}) \times E_{7}}=: d .
\end{aligned}
$$

\footnotetext{
${ }^{19}$ Available at http://www-math.univ-poitiers.fr/ maavl/.

${ }^{20} \mathrm{In}$ LiE, one first increases the maximal size by typing the command "maxobjects 99999999".
} 
By perusing the first few $a$ 's for the first few $p$ 's, one gets the following table: ${ }^{21}$

\begin{tabular}{|c|c|c|c|c|c|c|c|c|c|c|}
\hline$E_{7}, 56$ & $a=0$ & \begin{tabular}{ll|}
0 & 1 \\
\end{tabular} & 2 & 3 & 4 & 5 & $\sigma^{0}$ & & & 910 \\
\hline$p=2$ & $d=1$ & \begin{tabular}{ll|}
1 & 1 \\
\end{tabular} & 1 & 2 & 3 & 3 & 5 & & 7 & \begin{tabular}{l|l}
9 & 11
\end{tabular} \\
\hline$p=3$ & $d=1$ & $\begin{array}{lll}1 & 0 \\
\end{array}$ & 0 & 0 & 5 & 0 & 1 & & 6 & \\
\hline$p=4$ & $d=1$ & \begin{tabular}{ll|}
1 & 1 \\
\end{tabular} & 1 & 4 & 14 & 35 & & & & \\
\hline$p=5$ & $d=1$ & 10 & 0 & 0 & 31 & & & & & \\
\hline$p=6$ & $d=1$ & \begin{tabular}{|l|l|}
1 & 1 \\
\end{tabular} & 2 & 10 & & & & & & \\
\hline$p=7$ & $d=1$ & 10 & 2 & & & & & & & \\
\hline$p=8$ & $d=1$ & 11 & & & & & & & & \\
\hline
\end{tabular}

(throughout the treatment, the blank entries are seemingly not accessible with the computing facilities available to us.)

In the 2 -centered case $(p=2), \operatorname{dim} S_{1^{2}}(\mathbf{5 6})^{E_{7}}=1$ corresponds to $\mathcal{W}(2.17)$. The interpretation of the other results is as follows:

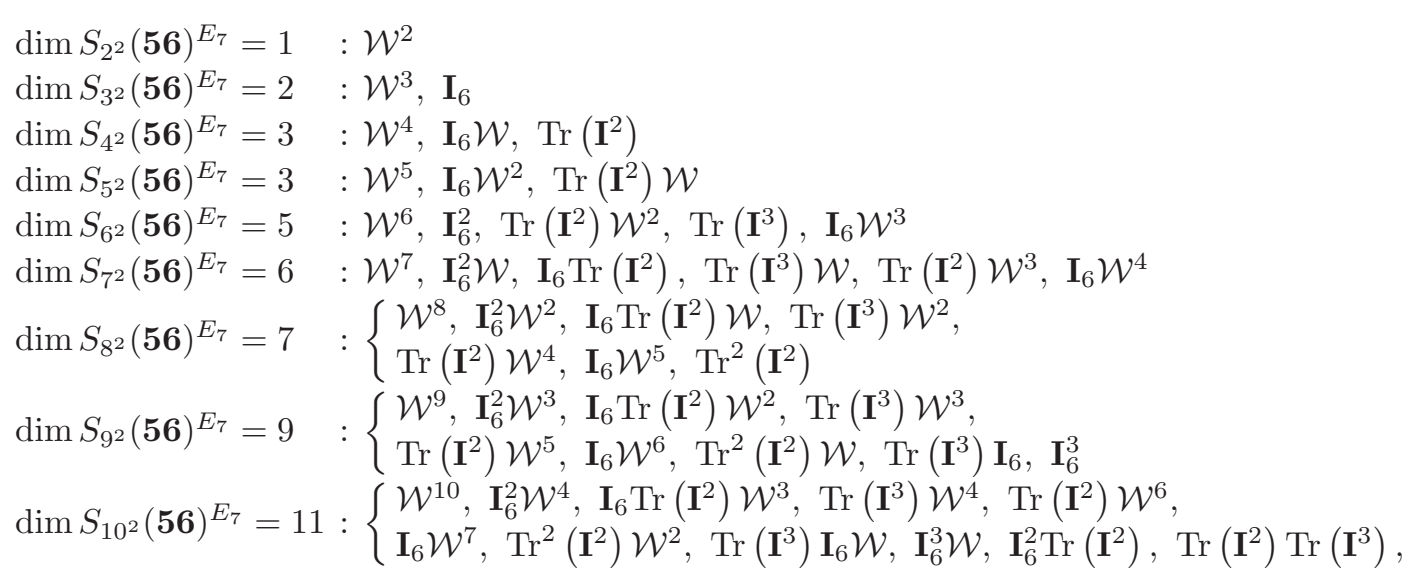

where the 2-centered polynomial invariants ${ }^{22} \mathbf{I}_{6}$ (degree 6), $\operatorname{Tr}\left(\mathbf{I}^{2}\right)$ (degree 8) and $\operatorname{Tr}\left(\mathbf{I}^{3}\right.$ ) (degree 12) have been firstly introduced in [48], and then studied in this very case in [35]. Note that no polynomial 2-centered invariants in the $\mathbf{5 6}$ of $E_{7}$ exist with an odd homogeneity degree, consistent with the observation made in section 2.2.2. The interpretation (2.32) of the $p=2$ row of table (2.31) is an evidence for the fact that the set

$$
\left\{\mathcal{W}, \mathbf{I}_{6}, \operatorname{Tr}\left(\mathbf{I}^{2}\right), \operatorname{Tr}\left(\mathbf{I}^{3}\right)\right\}
$$

is a complete basis for the ring of polynomial invariants of $(\mathbf{2}, \mathbf{5 6})$ of $S L_{h}(2, \mathbb{R}) \times E_{7}$, and it is finitely generating, namely all higher order polynomial invariants are simply polynomials in the polynomials of the set (2.33) itself [18].

In the 3 -centered case $(p=3)$, table $(2.31)$ yields that there are no $E_{7}$-invariants for the partitions $\lambda=1^{3}, 2^{3}, 3^{3}$ and hence there are no polynomial invariants of $(\mathbf{3}, \mathbf{5 6})$ of

\footnotetext{
${ }^{21}$ The result $\operatorname{dim}\left[S_{\lambda=0^{p}}(V)\right]^{G_{4}}=1$ always trivially refers to a numerical constant.

${ }^{22}$ As discussed at the end of section 3 of [35], for $p$ centers $\mathbf{I}_{6}$, as $\mathcal{W}(2.17)$, generally sits in the $\left(\wedge^{2} \mathbf{p}, \mathbf{1}\right)$ of $S L_{h}(p, \mathbb{R}) \times G_{4}$.
} 
$S L_{h}(3, \mathbb{R}) \times E_{7}$ with homogeneity degree $\leq 10$. The lowest possible degree is 12 , at which Lie finds 5 invariants. The absence of an invariant corresponding to the partition $\lambda=1^{3}$, i.e. of a " 3 -centered analogue" of $\mathcal{W}(2.17)$ can be explained by the fact that $\mathbf{1} \notin \mathbf{5 6} \mathbf{a}_{a}^{\otimes 3}$ (as mentioned, no invariant polynomials in the $\mathbf{5 6}$ of $E_{7}$ with an odd homogeneity degree exist at all). Then, one invariant of degree 18, and as many as 46 invariants of degree 24, are found.

In the 4-centered case $(p=4)$, there is an $E_{7}$-invariant of degree 4 (the lowest possible degree). It can be regarded as the "4-centered analogue" of $\mathcal{W}(2.17)$, whose existence can be explained by the fact that $\exists ! \mathbf{1} \in \mathbf{5} \mathbf{6}_{a}^{\otimes 4}$, given by the complete antisymmetrization of the product of two symplectic metrics $\mathbb{C}_{M N}$ of $\mathbf{5 6}$, such that $(a=1, \ldots, 4, M=1, \ldots, 56)$

$$
\operatorname{dim} S_{1^{4}}(\mathbf{5 6})^{E_{7}}=1: \mathcal{W}_{p=4}:=\frac{1}{4 !} \mathbb{C}_{[M N} \mathbb{C}_{P Q]} \epsilon^{a b c d} Q_{a}^{M} Q_{b}^{N} Q_{c}^{P} Q_{d}^{Q} .
$$

Thus, $\mathcal{W}_{p=4}(2.34)$ is the unique polynomial invariant of $(\mathbf{4}, \mathbf{5 6})$ of $S L_{h}(4, \mathbb{R}) \otimes E_{7}$ with homogeneity degree 4 . Its square yields the unique polynomial invariant of $(\mathbf{4}, \mathbf{5 6})$ of $S L_{h}(4, \mathbb{R}) \times E_{7}$ with homogeneity degree 8, as given by table $(2.31): \operatorname{dim} S_{2^{4}}(\mathbf{5 6})^{E_{7}}=1$.

In the 5 -centered case $(p=5)$, there are no invariants of degree $\leq 15$, since the partitions $\lambda=1^{5}, 2^{5}$ and $3^{5}$ do not yield any invariant for $E_{7}$. Once again, the absence of an invariant corresponding to the partition $\lambda=1^{5}$, i.e. of a "5-centered analogue" of $\mathcal{W}(2.17)$, can be explained by the fact that $\mathbf{1} \notin \mathbf{5} \boldsymbol{6}_{a}^{\otimes 5}$.

Finally, for the $p=6$ and 8 -centered cases, we see that there is a unique polynomial invariant of $(\mathbf{p}, \mathbf{5 6})$ of $S L_{h}(p, \mathbb{R}) \times E_{7}$ (corresponding to the partition $\lambda=1^{p}$ ); again, for $p=6$ and 8 it can be regarded as the " $p$-centered analogue" of $\mathcal{W}(2.17)$, whose existence can be explained by the fact that $\exists ! \mathbf{1} \in \mathbf{5} \boldsymbol{6}_{a}^{\otimes 6}$ and $\exists ! \mathbf{1} \in \mathbf{5} \boldsymbol{6}_{a}^{\otimes 8}$, given by the complete antisymmetrization of the product of $p=6,8$ symplectic metrics $\mathbb{C}_{M N}$ of $\mathbf{5 6}$, such that

$$
\begin{aligned}
& \operatorname{dim} S_{1^{6}}(\mathbf{5 6})^{E_{7}}=1: \mathcal{W}_{p=6}:=\frac{1}{6 !} \mathbb{C}_{[M N} \mathbb{C}_{P Q} \mathbb{C}_{R S]} \epsilon^{a b c d e f} Q_{a}^{M} Q_{b}^{N} Q_{c}^{P} Q_{d}^{Q} Q_{e}^{R} Q_{f}^{S} ; \\
& \operatorname{dim} S_{1^{8}}(\mathbf{5 6})^{E_{7}}=1: \mathcal{W}_{p=8}:=\frac{1}{8 !} \mathbb{C}_{[M N} \mathbb{C}_{P Q} \mathbb{C}_{R S} \mathbb{C}_{T U]} \epsilon^{a b c d e f g h} Q_{a}^{M} Q_{b}^{N} Q_{c}^{P} Q_{d}^{Q} Q_{e}^{R} Q_{f}^{S} Q_{g}^{T} Q_{h}^{U},
\end{aligned}
$$

where the "horizontal" $a$-indices range over $1, \ldots, 6$ and $1, \ldots, 8$ in $(2.35)$ and $(2.36)$, respectively.

\subsection{2 $G_{4}=\operatorname{Sp}(6, \mathbb{R}), R=\mathbf{1 4}^{\prime}$}

In supergravity, this is related to the $D=4$ theory with symmetric scalar manifold, based on the FTS $\mathfrak{M}\left(J_{3}^{\mathbb{R}}\right)$, namely the magic $\mathcal{N}=2$ Maxwell-Einstein theory over $J_{3}^{\mathbb{R}}$ (the rank-3 Euclidean Jordan algebras over the reals $\mathbb{R}[65-67])$.

In this case, the relevant $\operatorname{Sp}(6, \mathbb{R})$-representation is ${ }^{23} R=V\left(\lambda_{3}\right)=: \mathbf{1 4}^{\prime}$, namely the rank-3 completely antisymmetric skew-traceless representation, which is an irreducible

\footnotetext{
${ }^{23}$ There are actually two irreducible representations of $\operatorname{Sp}(6, \mathbb{R})$ with dimension 14 : the rank-2 antisymmetric skew-traceless $\mathbf{1 4}$, and the rank-3 antisymmetric skew-traceless $\mathbf{1 4}^{\prime}$; this latter characterizes $\operatorname{Sp}(6, \mathbb{R})$ as a group "of type $E_{7}$ " [27].
} 
component of $\wedge^{3} \mathbf{6}=: \mathbf{6}_{a}^{\otimes 3}$ (where $\mathbf{6}$ is the fundamental representation). The dimension $\operatorname{dim} S_{\left(a^{p}\right)}\left(\mathbf{1 4}^{\prime}\right)^{\mathrm{Sp}(6, \mathbb{R})}$ for the partition $\lambda=a^{p}$, yielding the (real) dimension of the vector space of polynomial invariants of homogeneity degree $p a$ in $p \operatorname{dim} R=14 p$ variables, is given as above:

$$
\operatorname{dim}\left[S_{\lambda=a^{p}}\left(\mathbf{1 4}^{\prime}\right)\right]^{\operatorname{Sp}(6, \mathbb{R})}=\operatorname{dim}\left[S^{p a}\left(\mathbf{p}, \mathbf{1 4}^{\prime}\right)\right]^{S L_{h}(p, \mathbb{R}) \times \operatorname{Sp}(6, \mathbb{R})}=: d .
$$

By perusing the first few $a$ 's for the first few $p$ 's, one gets the following table:

\begin{tabular}{|c|c|c|c|c|c|c|c|c|c|c|}
\hline $\operatorname{Sp}(6, \mathbb{R}), \mathbf{1 4}^{\prime}$ & $a=$ & \begin{tabular}{l|l}
0 & 1 \\
\end{tabular} & 2 & & & 5 & \begin{tabular}{l|l}
6 & 7
\end{tabular} & 8 & & 10 \\
\hline$p=2$ & $d=$ & 11 & 1 & & 3 & 3 & 56 & 7 & & 11 \\
\hline$p=3$ & $d=$ & 10 & 0 & & 1 & 0 & $\begin{array}{lll}0 & 0\end{array}$ & 3 & & \\
\hline$p=4$ & $d=$ & \begin{tabular}{l|l}
1 & 1
\end{tabular} & 2 & & & 28 & & & & \\
\hline$p=5$ & $d=$ & 10 & 0 & & 7 & & & & & \\
\hline$p=6$ & $d=$ & 11 & 2 & & & & & & & \\
\hline$p=7$ & $d=$ & 10 & 0 & & & & & & & \\
\hline$p=8$ & $d=$ & & 2 & & & & & & & \\
\hline
\end{tabular}

Considerations essentially analogous to the ones made for the case of $G_{4}=E_{7}$ and $R=\mathbf{5 6}$ hold in this case, and in subsequent cases, as well.

Note that the $p=2$ row of table (2.38) is identical to the $p=2$ row of table $(2.31)$; thus, the structure of the ring of polynomial invariants of $\left(\mathbf{2}, \mathbf{1 4}^{\prime}\right)$ of $S L_{h}(2, \mathbb{R}) \times \operatorname{Sp}(6, \mathbb{R})$ is the very same as the one of $(\mathbf{2}, \mathbf{5 6})$ of $S L_{h}(2, \mathbb{R}) \times E_{7}$. The same will hold for all other examples of groups "of type $E_{7}$ " relevant to $D=4$ supergravity which we will consider below, meaning that the structure of two-centered invariants, as well as their interpretation (2.32), is the very same in all these cases.

However, this does not hold any more already starting from the 3 -centered case $(p=3)$, as it is immediate to realize by comparing the $p=3$ rows of (2.31) and (2.38). Indeed, table (2.38), as table (2.31), yields that there are no $\operatorname{Sp}(6, \mathbb{R})$-invariants for the partitions $\lambda=1^{3}, 2^{3}, 3^{3}$ and hence there are no polynomial invariants of $\left(\mathbf{3}, \mathbf{1 4}^{\prime}\right)$ of $S L_{h}(3, \mathbb{R}) \times$ $\operatorname{Sp}(6, \mathbb{R})$ with homogeneity degree $\leq 10$, the lowest possible degree being 12 , at which however Lie finds 4 invariants, instead of 5 invariants as in the $E_{7}$ case treated above. As above, the absence of an invariant corresponding to the partition $\lambda=1^{3}$, i.e. of a "3-centered analogue" of $\mathcal{W}(2.17)$, can be explained by the fact that $\mathbf{1} \notin \mathbf{1} \mathbf{4}_{a}^{\prime \otimes 3}$.

2.3.3 $G_{4}=\mathrm{SO}(12), R=\mathbf{3 2}^{(\prime)}$

This is related to the $D=4$ theories with symmetric scalar manifold, based on the FTS's $\mathfrak{M}\left(J_{3}^{\mathbb{H}}\right)$ (magic $\mathcal{N}=2$ Maxwell-Einstein supergravity, sharing the same bosonic sector of $\mathcal{N}=6$ supergravity, both with $\left.G_{4}=S O^{*}(12)[65-67]\right)$ and $\mathfrak{M}\left(J_{3}^{\mathbb{H}_{s}}\right)$ (non-supersymmetric theory, with $\left.G_{4}=\mathrm{SO}(6,6)[74]\right)$, where $J_{3}^{\mathbb{H}}$ and $J_{3}^{\mathbb{H}_{s}}$ are rank-3 Euclidean Jordan algebras over the quaternions $\mathbb{H}$ and split quaternions $\mathbb{H}_{s}$, respectively.

In this case, the relevant $\mathrm{SO}(12)$-representation is $R=\mathbf{3 2}$ or $R=\mathbf{3 2}^{\prime}$, namely one of the two chiral spinor representations. The dimension $\operatorname{dim} S_{\left(a^{p}\right)}\left(\mathbf{3 2}^{(\prime)}\right)^{\mathrm{SO}(12)}$ for the partition $\lambda=a^{p}$, yielding the (real) dimension of the vector space of polynomial invariants of 
homogeneity degree $p a$ in $p \operatorname{dim} R=32 p$ variables, is given as above:

$$
\operatorname{dim}\left[S_{\lambda=a^{p}}\left(\mathbf{3 2}^{(\prime)}\right)\right]^{\mathrm{SO}(12)}=\operatorname{dim}\left[S^{p a}\left(\mathbf{p}, \mathbf{3 2}^{(\prime)}\right)\right]^{S L_{h}(p, \mathbb{R}) \times \mathrm{SO}(12)}=: d .
$$

By perusing the first few $a$ 's for the first few $p$ 's, one gets the following table:

\begin{tabular}{|c|c|c|c|c|c|c|c|c|c|c|}
\hline $\mathrm{SO}(12), \mathbf{3 2}^{(\prime)}$ & $a=$ & $\begin{array}{lll}01 \\
\end{array}$ & & 3 & 4 & 5 & & & & 10 \\
\hline$p=2$ & $d=$ & 11 & 1 & 2 & 3 & 3 & 5 & & & 11 \\
\hline$p=3$ & $d=$ & 10 & 0 & 0 & 5 & 0 & & & & \\
\hline$p=4$ & $d=$ & 111 & 2 & 5 & 17 & 42 & & & & \\
\hline$p=5$ & $d=$ & 10 & 0 & 0 & 42 & & & & & \\
\hline$p=6$ & $d=$ & 11 & 3 & 14 & & & & & & \\
\hline$p=7$ & $d=$ & 1 & 0 & & & & & & & \\
\hline$p=8$ & $d=$ & 11 & 4 & & & & & & & \\
\hline
\end{tabular}

Considerations essentially analogous to the ones made for the cases of $G_{4}=E_{7}, R=\mathbf{5 6}$ and $G_{4}=\operatorname{Sp}(6, \mathbb{R}), R=\mathbf{1 4}^{\prime}$ hold in this case, as well.

\subsection{4 $G_{4}=\mathrm{SU}(6), R=\mathbf{2 0}$}

This is related to the $D=4$ theories with symmetric scalar manifold, based on the FTS's $\mathfrak{M}\left(J_{3}^{\mathbb{C}}\right)$ (magic $\mathcal{N}=2$ Maxwell-Einstein theory over $J_{3}^{\mathbb{C}}$, with $G_{4}=\operatorname{SU}(3,3)[65-67]$ ) and $\mathfrak{M}\left(J_{3}^{\mathbb{C}_{s}}\right)$ (non-supersymmetric theory, with $G_{4}=\operatorname{SL}(6, \mathbb{R})[74]$ ), where $J_{3}^{\mathbb{C}}$ and $J_{3}^{\mathbb{C}_{s}}$ are rank-3 Euclidean Jordan algebras over the complex numbers $\mathbb{C}$ and split complex numbers $\mathbb{C}_{s}$, respectively. ${ }^{24}$

In this case, the relevant $\mathrm{SU}(6)$-representation is $R=\wedge^{3} \mathbf{6}=\mathbf{2 0}$, namely the rank-3 completely antisymmetric representation, built out from the fundamental representation 6. Due to the existence of the invariant $\epsilon$-tensor in the $\mathbf{6}$ of SU(6), the irrep. 20 is real. The dimension $\operatorname{dim} S_{\left(a^{p}\right)}(\mathbf{2 0})^{\mathrm{SU}(6)}$ for the partition $\lambda=a^{p}$, yielding the (real) dimension of the vector space of polynomial invariants of homogeneity degree $p a$ in $p \operatorname{dim} R=20 p$ variables, is given as above:

$$
\operatorname{dim}\left[S_{\lambda=a^{p}}(\mathbf{2 0})\right]^{\mathrm{SU}(6)}=\operatorname{dim}\left[S^{p a}(\mathbf{p}, \mathbf{2 0})\right]^{S L_{h}(p, \mathbb{R}) \times \mathrm{SU}(6)}=: d .
$$

\begin{tabular}{|c|c|c|c|c|c|c|c|c|c|c|}
\hline $\mathrm{SU}(6), \mathbf{2 0}$ & $a=$ & \begin{tabular}{|l|l|l}
0 & 1
\end{tabular} & & 3 & 4 & 5 & 6 & & & 10 \\
\hline$p=2$ & $d=$ & 11 & 1 & 2 & 3 & 3 & 5 & & & 11 \\
\hline$p=3$ & $d=$ & 10 & 1 & 0 & 5 & 0 & 9 & & & \\
\hline$p=4$ & $d=$ & 11 & 2 & 5 & 16 & 41 & & & & \\
\hline$p=5$ & $d=$ & 10 & $0 \mid 1$ & 0 & 37 & & & & & \\
\hline$p=6$ & $d=$ & \begin{tabular}{l|l}
1 & 1
\end{tabular} & 13 & 13 & & & & & & \\
\hline$p=7$ & $d=$ & 10 & $0 \mid 2$ & & & & & & & \\
\hline$p=8$ & $d=$ & 11 & $1 \mid 3$ & & & & & & & \\
\hline
\end{tabular}

By perusing the first few $a$ 's for the first few $p$ 's, one gets the following table:

Considerations essentially analogous to the previous cases hold in this case, as well.

\footnotetext{
${ }^{24}$ Actually, another supergravity theory exists in which $R=\mathbf{2 0}$, namely $\mathcal{N}=5, D=4$ supergravity, with $U$-duality group $G_{4}=\mathrm{SU}(1,5)$. However, this theory cannot be uplifted to $D=5$, and it is not related to a FTS, but rather to the Jordan triple system of $1 \times 2$ octonionic vectors $M_{1,2}(\mathbb{O})$ (see e.g. [65-67], and refs. therein).
} 
2.3.5 $G_{4}=\mathrm{SL}(2, \mathbb{R}) \times \mathrm{SL}(2, \mathbb{R}) \times \mathrm{SL}(2, \mathbb{R}), R=(\mathbf{2}, \mathbf{2}, \mathbf{2})$

We now consider the so-called $\mathcal{N}=2$ stu model $[49,50]$, whose $U$-duality group is $G_{4}=$ $\mathrm{SL}(2, \mathbb{R}) \times \mathrm{SO}(2,2) \cong \mathrm{SL}(2, \mathbb{R})^{3}$, with the relevant $\mathrm{BH}$ flux representation being the trifundamental $R=(\mathbf{2}, \mathbf{2}, \mathbf{2})$.

This provides an example of group "of type $E_{7}$ " [27] different from the ones treated above. Indeed, $\mathrm{SL}(2, \mathbb{R})^{3}$ can still be characterized as a conformal symmetry, but of a semisimple, rank-3 Jordan algebra, namely $J_{3}=\mathbb{R} \oplus \mathbb{R} \oplus \mathbb{R}$, or equivalently as the automorphism group of the FTS $\mathfrak{M}\left(J_{3}\right)$ constructed over such an algebra:

$$
\operatorname{SL}(2, \mathbb{R})^{3}=\operatorname{Conf}(\mathbb{R} \oplus \mathbb{R} \oplus \mathbb{R})=\operatorname{Aut}(\mathfrak{M}(\mathbb{R} \oplus \mathbb{R} \oplus \mathbb{R})) .
$$

Actually, by virtue of the isomorphism $\mathbb{R} \oplus \mathbb{R} \oplus \mathbb{R} \sim \mathbb{R} \oplus \Gamma_{1,1}$, this case can be regarded as the $(m, n)=(2,2)$ element of the infinite sequence of semi-simple rank-3 Jordan algebras $\mathbb{R} \oplus \Gamma_{m-1, n-1}$, where $\Gamma_{m-1, n-1}$ denotes the Clifford algebra of $O(m-1, n-1)$ [75]. This sequence can be related to $D=4$ supergravity theories (displaying symmetric scalar manifolds) for $m$ (or equivalently $n)=2(\mathcal{N}=2)$ or $6(\mathcal{N}=4)$. A complete basis of minimal degree (which turns out to be finitely generating [18]) of 2-centered BH invariant polynomials have been firstly determined in [48], and then further analyzed in [36] and [37].

The dimension $\operatorname{dim} S_{\left(a^{p}\right)}((\mathbf{2}, \mathbf{2}, \mathbf{2}))^{\mathrm{SL}(2, \mathbb{R})^{3}}$ for the partition $\lambda=a^{p}$, yielding the (real) dimension of the vector space of polynomial invariants of homogeneity degree $p a$ in $p \operatorname{dim} R=8 p$ variables, is given as above:

$$
\operatorname{dim}\left[S_{\lambda=a^{p}}((\mathbf{2}, \mathbf{2}, \mathbf{2}))\right]^{\operatorname{SL}(2, \mathbb{R})^{3}}=\operatorname{dim}\left[S^{p a}(\mathbf{p}, \mathbf{2}, \mathbf{2}, \mathbf{2})\right]^{S L_{h}(p, \mathbb{R}) \times \operatorname{SL}(2, \mathbb{R})^{3}}=: d .
$$

As done above, by perusing the first few $a$ 's for the first few $p$ 's, one gets the following

\begin{tabular}{|c|c|c|c|c|c|c|c|c|c|c|c|}
\hline $\mathrm{SL}(2, \mathbb{R})^{3}, \quad(\mathbf{2}, \mathbf{2}, \mathbf{2})$ & $a=$ & 01 & 2 & & 4 & 5 & 6 & 7 & 8 & 9 & 1 \\
\hline$p=2$ & $d=$ & $1]$ & & 4 & 7 & 9 & 14 & 17 & 2 & 2 & 3 \\
\hline$p=3$ & $d=$ & 10 & & 0 & 10 & 0 & 1 & 0 & 57 & 0 & 2 \\
\hline$p=4$ & $d=$ & 11 & 4 & 8 & 15 & 27 & & & & & \\
\hline$p=5$ & $d=$ & 10 & 0 & 0 & 10 & & & & & & \\
\hline$p=6$ & $d=$ & 1 & & 4 & & & & & & & \\
\hline$p=7$ & $d=$ & 1 & 0 & & & & & & & & \\
\hline$p=8$ & $d=$ & 1 & 1 & & & & & & & & \\
\hline
\end{tabular}
table:

We observe that the $p=2$ row of table (2.45) differs from the one of tables (2.31), (2.38), (2.40), (2.42), which instead all share the same row. This can be traced back to the semisimple nature of the rank-3 Jordan algebra $\mathbb{R} \oplus \Gamma_{1,1}$ to which the stu model is be related, to be contrasted to the simple rank-3 Jordan algebras corresponding to the cases treated above.

Moreover, it should be stressed that table (2.45) does not implement a peculiar symmetry of the stu model, namely the triality symmetry ${ }^{25}$ corresponding to the invariance

\footnotetext{
${ }^{25}$ The relevance of this symmetry to the theory of Quantum Information, and in particular to the classification of the quantum entanglement of three (and four) qubits has been recently studied, exploiting techniques and results from the supergravity side, also in the context of the so-called BH/qubit correspondence [56, 76-81].
} 
under the exchange of the three fundamentals 2's in $R=(\mathbf{2}, \mathbf{2}, \mathbf{2})$, achieved by imposing an invariance under the symmetric group $S_{3}$ acting on the three 2's in $R$.

The implementation of the triality symmetry will be explicitly worked out in section 4 for the case of $p=3$ and $a=4$, namely for the vector space of 3-centered invariant polynomials of degree 12, which, from table (2.45), has dimension 10; as yielded by the treatment of section 4.3.4, the dimension of the vector space of 3-centered invariant polynomials of degree 12 which are triality- (namely, $S_{3^{-}}$) symmetric, and thus relevant for black holes in the stu model, is 4 .

Our analysis can be refined as follows: by looking directly for the $\left(S L_{h}(2, \mathbb{R}) \times G_{4}\right)$ invariants as above, we now consider the $G_{4}$-invariants in $S^{k}\left(\left(\mathbb{R}^{2}\right) \otimes R\right)$. The formula (2.12) shows that these coincide with the $G_{4}$-invariants in $S_{\lambda}(R)$, tensored by the $S L_{h}(2, \mathbb{R})$ representation $S_{\lambda}\left(\mathbb{R}^{2}\right)$, where $\lambda \vdash k$ and $h t(\lambda) \leq 2$. By specifying this for the stu model, as done in all cases above, in Lie one types, for the partition $k=a+b$ with $a \geq b$, the following command (cfr. e.g. (2.29))

$$
\operatorname{plethysm}([a, b],[1,1,1], \mathrm{A} 1 \mathrm{~A} 1 \mathrm{~A} 1)[1] .
$$

As mentioned, if $d X[0,0,0]$ occurs in the output, the coefficient $d$ yields the dimension of the space of $G_{4}$-invariants in $S_{(a, b)}(R)$, otherwise there are no invariants in this representation.

In the 2-centered case $(p=2)$, an $S_{3}$-symmetric analysis of $\operatorname{SL}(2, \mathbb{R})^{3}$ - and $\left(S L_{h}(2, \mathbb{R}) \times \mathrm{SL}(2, \mathbb{R})^{3}\right)$ - invariant homogeneous polynomials for 2-centered BHs in the stu model has been performed in [36, 37, 48], whereas an $S_{4}$-symmetric treatment consistent in connection with the quantum entanglement of four qubits was given in [51].

Indeed, the relevant 2-centered representation for stu model is actually a quadrifundamental: for $p=2$ centers, one considers the invariants of the group $S L_{h}(2, \mathbb{R}) \times$ $\mathrm{SL}(2, \mathbb{R})^{3}$ in the representation $(\mathbf{2}, \mathbf{2}, \mathbf{2}, \mathbf{2})$. Thus, one may promote the $S_{3}$-invariance (triality) to an invariance (tetrality) under the symmetric group $S_{4}$ acting on the four fundamentals 2's in $(\mathbf{2}, \mathbf{2}, \mathbf{2}, \mathbf{2})$. A complete, minimal degree basis for the ring of $\left(S L_{h}(2, \mathbb{R}) \times \mathrm{SL}(2, \mathbb{R})^{3}\right)$ - invariant homogeneous polynomials is given by $\mathcal{W}$, together with 2 quartic polynomials and with a sextic one, denoted by ${ }^{26} \mathbf{I}_{6}^{\prime}[51]$.

When considering 2-centered BH physics, one must discriminate between the "horizontal" symmetry $S L_{h}(2, \mathbb{R})[48]$ and the $U$-duality symmetry $G_{4}=\operatorname{SL}(2, \mathbb{R})^{3}$, on which a triality must be implemented. Therefore, by down-grading $S_{4}$ (pertaining to four qubits in QIT) to $S_{3}$ (pertaining to 2-centered stu $\mathrm{BHs}$ ), the consistent $S_{3}$-invariant $p=2$ counting performed in $[36,37,48]$ yields that an invariant polynomial of degree 8 is no more generated by the previous ones, and a finitely generating [18] complete basis for the ring of $\left(S L_{h}(2, \mathbb{R}) \times \mathrm{SL}(2, \mathbb{R})^{3}\right)$ - invariant homogeneous polynomials is given by four elements of degree $2,4,6$ and 8 [48].

\footnotetext{
${ }^{26}$ Indeed, there is a slight difference in the definition of the $\left(S L_{h}(2, \mathbb{R}) \times G_{4}\right)$-invariant $\mathbf{I}_{6}$ for the models of $D=4$ (super)gravity based on simple $J_{3}$ 's [35] with respect to the definition of $\left(S L_{h}(2, \mathbb{R}) \times G_{4}\right)$-invariant $\mathbf{I}_{6}^{\prime}$ for the models of $D=4$ (super)gravity based on the semi-simple sequence $J_{3, m, n}:=\mathbb{R} \oplus \boldsymbol{\Gamma}_{m-1, n-1}[37,48]$; this is discussed in section 3 of [36].
} 


\section{Geometric interpretation}

In this section we consider the invariants for $S L_{h}(p) \times G_{4}$ in $\left(\mathbb{R}^{p}\right) \otimes R=:(\mathbf{p}, R)$ in the case that $^{27}$

$$
p \leq r:=\operatorname{dim} R \text {. }
$$

Note that $r$ is even whenever the symplectic invariant 2-form $\mathbb{C}_{M N}$ in $R_{a}^{\otimes 2}$ is non-degenerate (as we assume throughout the paper).

We start and recall some classical results (mainly referring to [52]), and then we discuss the associated geometrical interpretation in terms of Grassmannians.

The main result is the observation that the $G_{4}$-representation $S_{\left(a^{p}\right)}(R)$ which, as discussed in section 2, produces all invariants in $S^{a p}\left(\left(\mathbb{R}^{p}\right) \otimes R\right)$, can be identified with the representation of $G_{4}$ on the homogeneous polynomials of degree $a$ in the Plücker coordinates of the $p$-planes in $R$. Each of these Plücker coordinates is an $S L_{h}(p)$-invariant homogeneous polynomial of degree $p$ in the $p \operatorname{dim} R=p r$ coordinates on $\left(\mathbb{R}^{p}\right) \otimes R$. Thus, the $G_{4}$-invariant polynomials homogeneous of degree $a$ in these Plücker coordinates provide exactly the $\left(S L_{h}(p) \times G_{4}\right)$-invariant homogeneous polynomials of degree $a p$ which are the object of our investigation.

\subsection{Grassmannians}

\subsubsection{Invariants of $S L_{h}(p) \times G_{4}$ in $\left(\mathbb{R}^{p}\right) \otimes R$}

Any tensor $t$ in $\left(\mathbb{R}^{p}\right) \otimes R$ can be written as a sum $t=\sum_{a=1}^{\min (r, p)} x_{a} \otimes y_{a}$, with $x_{a} \in \mathbb{R}^{p}$, $y_{a} \in R$. Let $f_{1}, \ldots, f_{p}$ be the standard basis of $\mathbb{R}^{p}$. Writing each $x_{a}=\sum_{i=1}^{p} x_{a i} f_{i}$, and using the bilinearity of $\otimes$, one finds that

$$
t=\sum_{i=1}^{p} f_{i} \otimes r_{i}
$$

for certain uniquely determined elements $r_{i} \in R$.

Since any $\left(S L_{h}(p) \times G_{4}\right)$-invariant $F$ is obviously an $\left(S L_{h}(p) \times\{I\}\right)$-invariant, it is firstly convenient to study the invariants of $S L_{h}(p) \times\{I\}$. To this end, we only consider

\footnotetext{
${ }^{27}$ In the case $p>r$, one can easily show that there are no non-trivial invariants. This can be realized e.g. as follows.

One can write a tensor $t$ as $t=\sum_{i=1}^{p} f_{i} \otimes r_{i}$ (see eq. (3.2)). In the case $p>r$, it is however more convenient to choose a basis $e_{1}, \ldots, e_{r}$ of $R$, so that the same tensor can be rewritten as $t=\sum_{j=1}^{r} v_{j} \otimes e_{j}$, for (uniquely determined) vectors $v_{j} \in \mathbb{R}^{p}$.

For a generic $t$ (to be precise, for $t$ outside the closed subset of codimension $>1$ of $\mathbb{R}^{p} \otimes R$ defined by the vanishing of $r \times r$ minors of the matrix with rows $\left.v_{1}, \ldots, v_{r}\right)$, the vectors $v_{1}, \ldots, v_{r}$ are linearly independent. Thus, there exists an element $A \in S L_{h}(p, \mathbb{R})$ such that $A v_{i}=f_{i}$, where $\left\{f_{i}\right\}$ is the standard basis of $\mathbb{R}^{p}$. Therefore, under the action of $S L_{h}(p, \mathbb{R}) \times\{I\}$ all $t$ 's in a dense open subset of $\mathbb{R}^{p} \otimes R$ can be transformed into the 'standard' tensor $t=\sum_{j=1}^{r} f_{j} \otimes e_{j}$.

Consequently, there is only one orbit (on this dense open set); as any $\left(S L_{h}(p, \mathbb{R}) \times G_{4}\right.$ )-invariant polynomial must be constant on this orbit, such a polynomial must be a constant, and thus trivial. Note that in the limit case $r=p$, it could actually be given by the determinant of the matrix $\left(v_{1}, \ldots, v_{p}\right)$ (this is actually the unique invariant in the case $r=p$ ), but if $r<p$ then the codimension of the complement of this open orbit is $>1$, so a non-constant polynomial would be zero in one point and non-zero in another point of the open orbit, which yields a contradiction.
} 
the action of $S L_{h}(p)$ on the first factor of $\left(\mathbb{R}^{p}\right) \otimes R$, so we are actually dealing with the direct sum of $r$ copies of the fundamental representation $\mathbb{R}^{p}=: \mathbf{p}$ of $S L_{h}(p)$. In the case $r \geq p(3.1)$, the ring of invariants in this case is well understood. Fixing a basis $e_{1}, \ldots, e_{r}$ of $R$, this ring is generated by the determinants of the $(p \times p)$-minors of the $r \times p$ matrix $T:=T_{t}$ whose columns are the vectors $r_{1}, \ldots, r_{p}$ ([52], 11.1.2).

Note that all invariants $F$ vanish on the tensors $t=\sum_{i=1}^{p} f_{i} \otimes r_{i}$ such that the rank of the matrix $T_{t}$ is less than $p$, i.e. when the $r_{i}$ do not span a $p$-dimensional subspace of $R$; such tensors $t$ are called unstable (i.e., not semi-stable) tensors for this action. The (geometric) quotient $\left(\left(\mathbb{R}^{p}\right) \otimes R\right) / / S L_{h}(p)$ is the image of the quotient map $\pi$ given by generators of the ring of invariants $F([52], 11.1 .2)$ :

$$
\pi:\left(\mathbb{R}^{p}\right) \otimes R \longrightarrow \wedge^{p} R, \quad t=\sum_{i=1}^{p} f_{i} \otimes r_{i} \longmapsto r_{1} \wedge r_{2} \wedge \ldots \wedge r_{p} .
$$

Note that $\wedge^{p} R=: R_{a}^{\otimes p}=S_{\lambda}(R)$ (with partition $\lambda=1^{p}$ ) has basis $e_{I}=e_{i_{1}} \wedge \ldots \wedge e_{i_{p}}$, with $i_{1}<\ldots<i_{p}$, and therefore $\pi(t)=\sum t_{I} e_{I}$ (with $I$ collectively denoting the indices $i_{1}<\ldots<i_{p}$ ), where $t_{I}$ is the determinant of the minor of $T_{t}$ formed by the rows $i_{1}, \ldots, i_{p}$.

The image of the quotient map $\pi$ (3.3) consists of the decomposable tensors in $\wedge^{p} R$. This map, when restricted to stable points, is the lift to linear spaces of the Plücker map $G r(p, R) \rightarrow \mathbf{P}\left(\wedge^{p} R\right)$, where $G r(p, R)$ denotes the Grassmannian of $p$-planes in $R$ (see section 3.1.3).

Let now $F$ be an $\left(S L_{h}(p) \times G_{4}\right)$-invariant. Since it is trivially an $\left(S L_{h}(p) \times\{I\}\right)$ invariant, from the above reasoning $F$ is a polynomial in the determinants of $(p \times p)$-minors of $T_{t}$. Therefore, all such invariants can be determined with a two-step approach: ${ }^{28}$

1] first, one identifies the space of such polynomials as a representation of $G_{4}$;

2] then, one finds the $G_{4}$-invariants in that space.

Step 1 is actually well-known when one considers the space of such polynomials as a representation for the larger group $G L(R)=: G L(r)$ (namely, within (3.1)): as a $G L(R)$ representation, the space of polynomials, homogeneous of degree $a$ in the $(p \times p)$-minors of the $p \times r$ matrices, is $S_{a^{p}}(R)([52], 11.1 .2)$.

In order to find the $\left(S L_{h}(p) \times G_{4}\right)$-invariants in $\left(\mathbb{R}^{p}\right) \otimes R$, it then suffices to find the $G_{4}$-invariants in the representations $S_{a^{p}}(R)$ (step 2). This conclusion was already reached in section 2.2; however, the above discussion clarifies how a $G_{4}$-invariant in $S_{a^{p}}(R)$ produces a polynomial on $\left(\mathbb{R}^{p}\right) \otimes R$.

We are now going to reformulate this reasoning in a geometrical way.

\subsubsection{From tensors to planes}

In order to study $p$-centered BHs, for the case (3.1), one can use the Grassmannian $\operatorname{Gr}(p, R)$ of $p$-planes in $R$ as follows.

\footnotetext{
${ }^{28}$ It is funny to note that this approach is actually the opposite of the method which has been exploited in supergravity (especially in the 2-centered case $p=2$ ): in that framework, the $G_{4}$-invariants are organized in irreps. of $S L_{h}(p)$, from which one picks out the trivial (singlet) $S L_{h}(p)$-representations (see e.g. [35-37, 48]).
} 
Using the notation of section 3.1.1, any tensor $t$ in $\left(\mathbb{R}^{p}\right) \otimes R$ can be written as $t=$ $\sum_{i=1}^{p} f_{i} \otimes r_{i}$, for certain uniquely determined elements $r_{i} \in R$. It is here convenient to consider the dense open subset

$$
\left(\mathbb{R}^{p} \otimes R\right)^{0}:=\left\{\sum_{i=1}^{p} f_{i} \otimes r_{i}: \operatorname{dim}\left\langle r_{1}, \ldots, r_{p}\right\rangle=p\right\},
$$

such that the $p$ vectors $r_{1}, \ldots, r_{p}$ span a $p$-dimensional subspace of $R$ (the upperscript " 0 " denotes the absence of unstable points). This yields a map $\mathbf{G}$ to $G r(p, R)$ as follows:

$$
\mathbf{G}:\left(\left(\mathbb{R}^{p}\right) \otimes R\right)^{0} \longrightarrow G r(p, R), \quad t=\sum_{i=1}^{p} f_{i} \otimes r_{i} \longmapsto W_{t}:=\left\langle r_{1}, \ldots, r_{p}\right\rangle .
$$

It is worth noting that the action of $S L_{h}(p)$ on $\mathbb{R}^{p}$ merely changes the basis of $W_{t}$, so the map $\mathbf{G}$ is $S L_{h}(p)$-invariant. It is obviously also $G L_{h}(p)$-invariant, so it is actually identifying more tensors than strictly necessary for our purposes. The map $\mathbf{G}$ (3.5), besides being injective, is obviously also surjective: indeed, given a $p$-plane $W \subset R$, one can choose a basis $r_{1}, \ldots, r_{p}$, and then $W=W_{t}$, where $t=\sum_{i=1}^{p} f_{i} \otimes r_{i}$. Thus, one gets the following bijection

$$
\left(\left(\mathbb{R}^{p}\right) \otimes R\right)^{0} / G L_{h}(p) \longleftrightarrow G r(p, R), \quad t \longleftrightarrow W_{t} .
$$

In particular, any $G_{4}$-invariant function on the Grassmannian $G r(p, R)$ of $p$-planes in $R$ will yield an $\left(S L_{h}(p) \times G\right)$-invariant function on $\left(\left(\mathbb{R}^{p}\right) \otimes R\right)^{0}$, which will eventually extend ${ }^{29}$ to the whole relevant irrep. $\left(\mathbb{R}^{p}\right) \otimes R$.

\subsubsection{The Plücker map}

As $\operatorname{Gr}(p, R)$ is (a real subset of) a projective variety, which is moreover a $p(r-p)$ dimensional homogeneous space:

$$
G r(p, r) \cong \frac{O(r)}{O(p) \otimes O(r-p)},
$$

one can proceed as follows. Recall that the Plücker map $\mathcal{P}$ is defined as the embedding

$$
\mathcal{P}: G r(p, R) \longrightarrow \mathbf{P}\left(\wedge^{p} R\right), \quad W_{t} \longmapsto \wedge^{p} W_{t} .
$$

In particular, the composition $\mathcal{P} \circ \mathbf{G}$ of this map with $\mathbf{G}(3.5)$ maps $t$ to $r_{1} \wedge \ldots \wedge r_{p}$. Fixing a basis $e_{1}, \ldots, e_{r}$ of $R$, one thus gets the basis $e_{I}=e_{i_{1}} \wedge \ldots \wedge e_{i_{p}}$, with $i_{1}<\ldots<i_{p}$, of $\wedge^{p} R$ (cfr. below (3.3)). The Plücker coordinates of $W_{t}$ are defined as the $(p \times p)$-minors of the $r \times p$ matrix $T:=T_{t}$ with columns $r_{1}, \ldots, r_{p}$.

The action of the group $G L_{h}(R)$ can be represented on the space of global sections $\Gamma(G r(p, R), L)$ on a line bundle $L$ over $G r(p, R)$. Working over the complex numbers and denoting by Pic $(X)$ the Picard group of the variety $X$, let us recall that $\operatorname{Pic}(\operatorname{Gr}(p, R))$ is generated by a (very ample) line bundle $L$, whose global sections are the Plücker coordinates

\footnotetext{
${ }^{29}$ In the present investigation, as resulting from section 2, we consider homogeneous polynomial invariants; in such a case, the extension from $\left(\left(\mathbb{R}^{p}\right) \otimes R\right)^{0}$ to the whole $\left(\mathbb{R}^{p}\right) \otimes R$ is immediate.
} 
themselves. In fact, $\Gamma(\operatorname{Gr}(p, R), L) \cong \wedge^{p} R$, (actually the dual representation thereof, since the coordinates are linear maps on $\wedge^{p} R$ ). The action of $G L_{h}(R)$ on $R$ then induces an action on the Grassmannian $G r(p, R)$ and thus on the spaces of global sections $\Gamma(G r(p, R), L)$. By recalling that $\wedge^{p} R=S_{\lambda}(R)$ with partition $\lambda=1^{p}$ (cfr. below (2.6)), Bott's theorem (see e.g. [53]) gives, as $G L_{h}(R)$-representations:

$$
\Gamma\left(G r(p, R), L^{\otimes a}\right) \cong S_{a \lambda}(R), \quad a \lambda:=\underbrace{(a, \ldots, a)}_{p}=: a^{p} .
$$

Furthermore, any global section of $L^{\otimes a}$ is a linear combination of products of a sections of $L$ (and therefore the map $S^{a} \Gamma(L) \rightarrow \Gamma\left(L^{\otimes a}\right)$ is surjective); in terms of representations, this simply amounts to the statement that $S_{a \lambda}$ is a summand of $S^{a p}(R)$. Thus, any section of $L^{\otimes a}$ is a homogeneous polynomial in the Plücker coordinates of degree $a$.

Given a $G_{4}$-invariant $F \in S_{a \lambda}(R) \cong \Gamma\left(G r(p, R), L^{\otimes a}\right)$, it corresponds to a degree $a$ homogeneous polynomial in the Plücker coordinates, defined by the map (recall (3.5) and (3.6)):

$$
F: G r(p, R) \longrightarrow \mathbb{R}
$$

Thus, the composition

$$
F \circ \mathbf{G}:\left(\left(\mathbb{R}^{p}\right) \otimes R\right)^{0} \longrightarrow \wedge^{p} R \longrightarrow \mathbb{R}
$$

yields a $\left(S L_{h}(p) \times G_{4}\right)$-invariant which extends to the whole $\left(\mathbb{R}^{p}\right) \otimes R$. This provides a geometrical explanation of the treatment of section 2, and in particular of the fact that the $S_{\lambda}(R)$ with $\lambda=a^{p}$ contribute to - and actually are the unique responsible for - the $\left(S L_{h}(p) \times G_{4}\right)$-invariant homogeneous polynomials in $\left(\mathbb{R}^{p}\right) \otimes R$.

To summarize, in order to find $\left(S L_{h}(p) \otimes G_{4}\right)$-invariant homogeneous polynomials $F$ in the representation $\left(\mathbb{R}^{p}\right) \otimes R$, one needs to find invariant polynomials $\hat{F}$ for the induced action of $G_{4}$ on $\wedge^{p} R$ :

$$
F(t)=\hat{F}\left(\ldots, p_{i_{1} \ldots i_{p}}(t), \ldots\right)
$$

where $p_{i_{1} \ldots i_{p}}(t)=p_{\left[i_{1} \ldots i_{p}\right]}(t)$.

In particular, if an invariant $F$ is a homogeneous polynomial of degree $k$ in the coefficients $c_{i j}$ of $t=\sum c_{i j} f_{i} \otimes e_{j}$, then, as each Plücker coordinate is homogeneous of degree $p$ in the $c_{i j}, \hat{F}$ is homogeneous of degree $k / p$ in the Plücker coordinates. Thus, $k$ must be a multiple of $p$. This matches the statement made below (2.15), and it is not surprising, as $\operatorname{SL}(p, \mathbb{C})$ contains the diagonal matrices $\omega I$ where $\omega=e^{2 \pi i / p}$ and these act by multiplication by $\omega^{d}$ on polynomials $F$ of degree $k$; so, if $F$ is $S L_{h}(p)$-invariant, $k$ must indeed be a multiple of $p$. Moreover, these invariants $\hat{F}$ should be non-zero when restricted to the (semi-)stable decomposable tensors.

\section{3-centered stu black holes}

We will now apply the method discussed in sections 2 and 3 to compute the invariants pertaining to 3 -centered $(p=3) \mathrm{BHs}$ in the $\mathcal{N}=2, D=4$ stu model $[49,50]$. As discussed 
in section 2.3.5, in this case the $U$-duality group is $G_{4}=\mathrm{SL}(2, \mathbb{R}) \times \mathrm{SO}(2,2) \cong \mathrm{SL}(2, \mathbb{R})^{3}$, with the relevant $\mathrm{BH}$ representation being the tri-fundamental $R=(\mathbf{2}, \mathbf{2}, \mathbf{2})$. Moreover, the $K$-tensor (namely, the unique rank-4 symmetric invariant in $(\mathbf{2}, \mathbf{2}, \mathbf{2})_{s}^{\otimes 4}$; see section 1) is given by the Cayley's hyperdeterminant on $R$ [54-56].

In table (2.45), we have computed the dimension of the spaces of invariants for $p=3$ up to degree 30 . In particular, the lowest degree non-trivial $\left(S L_{h}(3, \mathbb{R}) \times S L(2, \mathbb{R})^{3}\right)$ invariant homogeneous polynomials in the $\mathbb{R}^{3} \otimes(\mathbf{2}, \mathbf{2}, \mathbf{2})=:(\mathbf{3}, \mathbf{2}, \mathbf{2}, \mathbf{2})$ have degree 12 , and they span a 10-dimensional space.

From the treatment of sections 2 and 3, as well as from table (2.45), such 3-centered invariant polynomials lie in $S_{4^{3}}((\mathbf{2}, \mathbf{2}, \mathbf{2}))$. In the present section, we will determine a basis for their 10-dimensional space. Then, in subsubsection 4.3.4 we will implement invariance (triality) under the $S_{3}$ symmetric group acting on the three 2's in $R$, obtaining a basis of the resulting 4-dimensional vector space of $\left(S_{3} \times S L_{h}(3, \mathbb{R}) \times S L(2, \mathbb{R})^{3}\right)$-invariant homogeneous polynomials of degree 12 in the $(\mathbf{3}, \mathbf{2}, \mathbf{2}, \mathbf{2})$, thus pertaining to the description of 3-centered BHs in the stu model.

\subsection{Invariant from Cayley's hyperdeterminant: $S^{4}\left(S_{1^{3}}((\mathbf{2}, \mathbf{2}, \mathbf{2}))\right)$}

A first invariant can be constructed as follows.

Let us recall that the $\mathrm{BH}$ flux irrep. $R=(\mathbf{2}, \mathbf{2}, \mathbf{2})$ is endowed with an invariant alternating form $\mathbb{C}$, i.e. the symplectic $8 \times 8$ metric $\mathbb{C}_{M N}:=(\exists !) \mathbf{1} \in(\mathbf{2}, \mathbf{2}, \mathbf{2})_{a}^{\otimes 2}$. Within the notation of section 3.1, the restriction of $\mathbb{C}_{M N}$ to the 3 -dimensional subspace $W_{t} \subset(\mathbf{2}, \mathbf{2}, \mathbf{2})$ generated by 3 given charge vectors $Q_{i}=: r_{i} \in(\mathbf{2}, \mathbf{2}, \mathbf{2})$ (we here denote the "horizontal" index as $i=1,2,3=p$ ) is given by the $3 \times 3$ alternating matrix

$$
\mathbb{C}_{t}:=\left.\mathbb{C}\right|_{W_{t} \otimes W_{t}}=\left(\begin{array}{ccc}
0 & \left(r_{1}, r_{2}\right) & \left(r_{1}, r_{3}\right) \\
\left(r_{2}, r_{1}\right) & 0 & \left(r_{2}, r_{3}\right) \\
\left(r_{3}, r_{1}\right) & \left(r_{3}, r_{2}\right) & 0
\end{array}\right), \quad W_{t}:=\left\langle r_{1}, r_{2}, r_{3}\right\rangle \subset(\mathbf{2}, \mathbf{2}, \mathbf{2}),
$$

where $(\operatorname{cfr} .(2.17) ; M=1, \ldots, 8=\operatorname{dim}(\mathbf{2}, \mathbf{2}, \mathbf{2}))$

$$
\left(\mathbb{C}_{t}\right)_{i j}=\left(r_{i}, r_{j}\right):=\mathbb{C}_{M N} r_{i}^{M} r_{j}^{N}=: \mathcal{W}_{i j}=-\mathcal{W}_{j i}
$$

is the $S L(2, \mathbb{R})^{3}$-invariant symplectic product of $r_{i}$ and $r_{j}$. It is immediate to realize that $\mathcal{W}_{i j}(i, j=1,2,3)$ belongs to the $\mathbf{3}^{\prime}=\wedge^{2} \mathbf{3}$ of $S L_{h}(3, \mathbb{R})$ (cfr. end of section 2.2.1, as well as the end of section 3 of [35]); indeed, by using the Ricci-Levi-Civita invariant symbol $\epsilon^{i j k}$ of $S L_{h}(3, \mathbb{R})$, one can define

$$
\mathcal{W}^{i}:=\frac{1}{2} \epsilon^{i j k} \mathbb{C}_{M N} r_{j}^{M} r_{k}^{N}=\frac{1}{2} \epsilon^{i j k} \mathcal{W}_{j k} \in\left(\mathbf{3}^{\prime}, \mathbf{1}, \mathbf{1}, \mathbf{1}\right) \text { of } S L_{h}(3, \mathbb{R}) \times S L(2, \mathbb{R})^{3} .
$$

The vector

$$
v_{t}:=\left(r_{2}, r_{3}\right) r_{1}+\left(r_{3}, r_{1}\right) r_{2}+\left(r_{1}, r_{2}\right) r_{3}=\frac{1}{2} \epsilon^{i j k} \mathcal{W}_{j k} r_{i}=\frac{1}{2} \epsilon^{i j k} \mathcal{W}_{[j k} r_{i]} \in W_{t}
$$

spans the kernel of $\mathbb{C}_{t}(4.1)$, and it can be considered as a multilinear alternating map

$$
v_{t}:(\mathbf{2}, \mathbf{2}, \mathbf{2})^{\otimes 3} \longrightarrow(\mathbf{2}, \mathbf{2}, \mathbf{2}), \quad\left(r_{1}, r_{2}, r_{3}\right) \longmapsto\left(r_{2}, r_{3}\right) r_{1}+\left(r_{3}, r_{1}\right) r_{2}+\left(r_{1}, r_{2}\right) r_{3} .
$$


In order to see this, it suffices to check that it is alternating for the permutations (12) and (23), which is easily done. Thus, the map $v_{t}(4.5)$ induces a linear map $\wedge^{3}(\mathbf{2}, \mathbf{2}, \mathbf{2}) \rightarrow$ $(\mathbf{2}, \mathbf{2}, \mathbf{2})$; by virtue of the treatment of section 3 , this proves that $v_{t}$ is a linear combination of the $r_{i}$ with coefficients which are linear forms in the Plücker coordinates of $t$. From the treatment of section 3, these Plücker coordinates are homogeneous of degree $p=3$ in the coordinates $c_{i j}$ of $t$, and they are invariant under the action of $S L_{h}(3, \mathbb{R})$, hence

$$
v_{t}=v_{(A, I) t}, \quad \forall A \in S L_{h}(3, \mathbb{R}),
$$

implying that

$$
v_{t} \in\left(\mathbf{1}, W_{t}\right) \subset(\mathbf{1}, \mathbf{2}, \mathbf{2}, \mathbf{2}) .
$$

As the symplectic 2 -form $\mathbb{C}$ is $S L(2, \mathbb{R})^{3}$-invariant, by recalling definition (4.4) one obtains the following formula for the action of $B \in S L(2, \mathbb{R})^{3}$ on $v_{t}$ itself:

$$
\begin{aligned}
B v_{t} & =\left(r_{2}, r_{3}\right) B r_{1}+\left(r_{3}, r_{1}\right) B r_{2}+\left(r_{1}, r_{2}\right) B r_{3} \\
& =\left(B r_{2}, B r_{3}\right) B r_{1}+\left(B r_{3}, B r_{1}\right) B r_{2}+\left(B r_{1}, B r_{2}\right) B r_{3} \\
& =v_{(I, B) t} .
\end{aligned}
$$

By virtue of (4.6), since

$$
v_{(A, B) t}=v_{(A, I)(I, B) t}=v_{(I, B) t}=B v_{t}
$$

any $S L(2, \mathbb{R})^{3}$-invariant polynomial $F$ of degree $g$ on the tri-fundamental representation $R=(\mathbf{2}, \mathbf{2}, \mathbf{2})$ produces an $\left(S L_{h}(3, \mathbb{R}) \times S L(2, \mathbb{R})^{3}\right)$-invariant polynomial $F_{0}$ homogeneous of degree $3 g$ on $(\mathbf{3}, \mathbf{2}, \mathbf{2}, \mathbf{2})$, defined as follows:

$$
F_{0}(t):=F\left(v_{t}\right)
$$

A natural choice is $F=\mathcal{I}_{4}$, where $\mathcal{I}_{4}$ is the Cayley's hyperdeterminant $[54,55]$ on $(\mathbf{2}, \mathbf{2}, \mathbf{2})$ (determined by the $K$-tensor of $(\mathbf{2}, \mathbf{2}, \mathbf{2})[54-56])$; this is an homogeneous polynomial of degree 4 , and it is the unique algebraically independent $S L(2, \mathbb{R})^{3}$ invariant polynomial on the $(\mathbf{2}, \mathbf{2}, \mathbf{2})$ itself. Therefore, the choice $F=\mathcal{I}_{4}$ yields an $\left(S L_{h}(3, \mathbb{R}) \times S L(2, \mathbb{R})^{3}\right)$-invariant polynomial $F_{0}$ homogeneous of degree $3 \cdot 4=12$ for 3 -centered BHs in the stu model:

$$
F_{0}(t):=\mathcal{I}_{4}\left(v_{t}\right)
$$

The construction performed above can be clarified in terms of representation theory as follows.

From the treatment of sections 2 and 3 (in particular, recalling (3.12)), the $\left(S L_{h}(3, \mathbb{R}) \otimes G_{4}\right)$-invariants homogeneous polynomials $F$ on $\left(\mathbb{R}^{3}\right) \otimes R=:(\mathbf{3}, R)$ are given by invariants $\hat{F}$ for the induced action of $G_{4}$ on $\wedge^{3} R=: R_{a}^{\otimes 3}=S_{\lambda}(R)$ (with partition $\lambda:=1^{3}$; see below $\left.(2.6)\right)$ :

$$
F(t)=\hat{F}\left(\ldots, p_{i_{1} i_{2} i_{3}}(t), \ldots\right)
$$

(where $p_{i_{1} i_{2} i_{3}}(t)=p_{\left[i_{1} i_{2} i_{3}\right]}(t)$ ) which should be non-zero when restricted to the (semi-)stable decomposable tensors. 
In general, the representations of $G_{4}$ on $\wedge^{3} R$ may be reducible. Indeed, for the stu model we have

$$
S_{1^{3}}((\mathbf{2}, \mathbf{2}, \mathbf{2})):=\wedge^{3}(\mathbf{2}, \mathbf{2}, \mathbf{2}) \equiv(\mathbf{2}, \mathbf{2}, \mathbf{2})_{a}^{\otimes 3} \cong(\mathbf{2}, \mathbf{2}, \mathbf{2}) \oplus(\mathbf{4}, \mathbf{2}, \mathbf{2}) \oplus(\mathbf{2}, \mathbf{4}, \mathbf{2}) \oplus(\mathbf{2}, \mathbf{2}, \mathbf{4}),
$$

where 4 denotes the spin $s=3 / 2$ irrep. of $\mathrm{SL}(2, \mathbb{R})$.

The appearance of $(\mathbf{2}, \mathbf{2}, \mathbf{2})$ in the r.h.s. of $(4.13)$, and in general the fact that $R \in R_{a}^{\otimes 3}$, can be simply related to the existence of the $G_{4}$-equivariant map

$$
R \longrightarrow \wedge^{3} R, \quad r \longmapsto \mathbb{C}^{*} \wedge r
$$

where $\mathbb{C} \in \wedge^{2} R^{*}$ corresponds to $\mathbb{C}^{*} \in \wedge^{2} R$ under the duality given by the non-degenerate symplectic form $\mathbb{C} \equiv \mathbb{C}_{M N}=\mathbb{C}_{[M N]}$ on $R$ (symplectic structure of - generalized - electricmagnetic duality in $D=4$ ). This implies that any $G_{4}$-invariant on $R$ trivially produces a $G_{4}$-invariant on $\wedge^{3} R$.

Let us call $\Psi_{t}$ the generalization (for a generic case) of the map $v_{t}(4.4)-(4.5)$ constructed above:

$$
\Psi_{t}: \wedge^{3} R \longrightarrow R, \quad r_{1} \wedge r_{2} \wedge r_{3} \longmapsto\left(r_{2}, r_{3}\right) r_{1}+\left(r_{3}, r_{1}\right) r_{2}+\left(r_{1}, r_{2}\right) r_{3}
$$

which then satisfies (cfr. (4.8))

$$
\Psi_{t}\left(B\left(r_{1} \wedge r_{2} \wedge r_{3}\right)\right):=\Psi_{t}\left(\left(B r_{1}\right) \wedge\left(B r_{2}\right) \wedge\left(B r_{3}\right)\right)=B \Psi_{t}\left(r_{1} \wedge r_{2} \wedge r_{3}\right), \forall B \in G_{4},
$$

since $\left(B r_{i}, B r_{j}\right)=\left(r_{i}, r_{j}\right)$. Thus the map $\Psi_{t}$ (4.15) is, up to scalar multiplication, the unique $G_{4}$-equivariant projection of $\wedge^{3} R$ onto $R$.

Thus, coming back to the stu model, it follows that, up to a real scalar, the map $\pi:(\mathbf{3}, \mathbf{2}, \mathbf{2}, \mathbf{2}) \rightarrow \wedge^{3}(\mathbf{2}, \mathbf{2}, \mathbf{2})$ (cfr. (3.3) for $\left.p=3\right)$ is given by

$$
\pi(t)=v_{t}+w_{t}, \quad v_{t}:=\Psi_{t}\left(W_{t}\right) \in(\mathbf{2}, \mathbf{2}, \mathbf{2}), \quad w_{t} \in(\mathbf{4}, \mathbf{2}, \mathbf{2}) \oplus(\mathbf{2}, \mathbf{4}, \mathbf{2}) \oplus(\mathbf{2}, \mathbf{2}, \mathbf{4}) .
$$

This leads to the invariant $F_{0}(4.11)$, which is thus given by the image of $S^{4}\left(S_{1^{3}}(\mathbf{2}, \mathbf{2}, \mathbf{2})\right)$ in $S_{4,4,4}((\mathbf{2}, \mathbf{2}, \mathbf{2}))$.

From the treatment above, it clearly follows that the degree-12 homogeneous $\left(S L_{h}(3, \mathbb{R}) \times G_{4}\right)$-invariant polynomial $F_{0}(4.11)$ can be consistently defined for all groups $G_{4}$ "of type $E_{7}$ ", and in particular at least for the class relevant to $D=4$ supergravity theories with symmetric scalar manifolds, listed in table 1 .

\subsection{Other invariants from $S^{2}\left(S_{2^{3}}((2,2,2))\right)$}

As a natural next step, one can try to determine other $S L(2, \mathbb{R})^{3}$-invariants of degree 12 from quadratic invariants in $S_{2^{3}}((\mathbf{2}, \mathbf{2}, \mathbf{2}))$.

Using LiE, one can decompose $S_{2^{3}}((\mathbf{2}, \mathbf{2}, \mathbf{2}))$ into irreducible $S L(2, \mathbb{R})^{3}$ representations:

$$
S_{2^{3}}((\mathbf{2}, \mathbf{2}, \mathbf{2})) \cong(\mathbf{3}, \mathbf{1}, \mathbf{1})^{\oplus 3} \oplus(\mathbf{1}, \mathbf{3}, \mathbf{1})^{\oplus 3} \oplus(\mathbf{1}, \mathbf{1}, \mathbf{3})^{\oplus 3} \oplus \ldots,
$$


where the dots denote other 25 terms, which are not relevant for our purposes. 3 denotes the adjoint ( $\operatorname{spin} s=1$ ) irrep. of $\mathrm{SL}(2, \mathbb{R})$, which has a unique quadratic invariant (the $\mathrm{SL}(2, \mathbb{R}) \sim \mathrm{SO}(2,1)$ Cartan-Killing invariant metric $\eta=\operatorname{diag}(1,1-1))$; as a consequence, since $\mathbf{1}$ denotes the singlet, there is a unique quadratic invariant induced onto the $(\mathbf{3}, \mathbf{1}, \mathbf{1})$, $(\mathbf{1}, \mathbf{3}, \mathbf{1})$ and $(\mathbf{1}, \mathbf{1}, \mathbf{3})$ of $S L(2, \mathbb{R})^{3}$. Thus, from the representations in the r.h.s. of (4.18), one obtains $3 \cdot 3=9$ quadratic $S L(2, \mathbb{R})^{3}$-invariant structures:

$$
\begin{aligned}
& \exists !(\mathbf{1}, \mathbf{1}, \mathbf{1}) \in(\mathbf{3}, \mathbf{1}, \mathbf{1}) \otimes_{s}(\mathbf{3}, \mathbf{1}, \mathbf{1}) \quad(3 \text { times }) ; \\
& \exists !(\mathbf{1}, \mathbf{1}, \mathbf{1}) \in(\mathbf{1}, \mathbf{3}, \mathbf{1}) \otimes_{s}(\mathbf{1}, \mathbf{3}, \mathbf{1}) \quad(3 \text { times }) \text {; } \\
& \exists !(\mathbf{1}, \mathbf{1}, \mathbf{1}) \in(\mathbf{1}, \mathbf{1}, \mathbf{3}) \otimes_{s}(\mathbf{1}, \mathbf{1}, \mathbf{3}) \quad(3 \text { times }) .
\end{aligned}
$$

One can check that these 9 invariants, together with $F_{0}$ (4.11), yield 10 linearly independent invariants in $S_{4^{3}}((\mathbf{2}, \mathbf{2}, \mathbf{2}))$. Thus, as announced, they do provide a complete basis for the 10-dimensional space of $\left(S L_{h}(3, \mathbb{R}) \times S L(2, \mathbb{R})^{3}\right)$-invariant homogeneous polynomials of degree 12 in the $(\mathbf{3}, \mathbf{2}, \mathbf{2}, \mathbf{2})$, as resulting from table $(2.45)$.

\subsection{Explicit construction}

Let $f_{1}, f_{2}, f_{3}$ and $e_{1}, \ldots, e_{8}$ be the standard basis of $\mathbb{R}^{3}=: \mathbf{3}$ of $S L_{h}(3, \mathbb{R})$, and of $(\mathbf{2}, \mathbf{2}, \mathbf{2})$ of $S L(2, \mathbb{R})^{3}$, respectively. Thus, any tensor $t \in(\mathbf{3}, \mathbf{2}, \mathbf{2}, \mathbf{2})$ of $S L_{h}(3, \mathbb{R}) \times S L(2, \mathbb{R})^{\otimes 3}$ can be written $\operatorname{as}^{30}$ (using the notation of section (3.1.1), and in particular denoting the "horizontal" index by $i=1,2,3$ )

$$
t=\sum_{i=1,2,3, j=1, \ldots, 8} c_{i j} f_{i} \otimes e_{j}=\sum_{i=1}^{3} f_{i} \otimes r_{i},
$$

for certain uniquely determined elements $r_{i} \in(\mathbf{2}, \mathbf{2}, \mathbf{2})$ of $S L(2, \mathbb{R})^{3}$.

As discussed in section 3 , the Plücker coordinates $p_{i_{1} i_{2} i_{3}}(t)$ of the tensor $t$ are the determinants of the $3 \times 3$ matrices formed by the the rows $i_{1}, i_{2}, i_{3}$ of the $8 \times 3$ matrix which has columns $r_{1}, r_{2}, r_{3}$ :

$$
p_{i_{1} i_{2} i_{3}}(t)=\operatorname{det}\left(\begin{array}{lll}
c_{1 i_{1}} & c_{2 i_{1}} & c_{3 i_{1}} \\
c_{1 i_{2}} & c_{2 i_{2}} & c_{3 i_{2}} \\
c_{1 i_{3}} & c_{2 i_{3}} & c_{3 i_{3}}
\end{array}\right) \text {. }
$$

This is the formula defining $p_{i_{1} i_{2} i_{3}}=p_{\left[i_{1} i_{2} i_{3}\right]}$, and their number is indeed $\left(\begin{array}{l}8 \\ 3\end{array}\right)=56$.

In the stu model $G_{4}=S L(2, \mathbb{R})^{3}$, with Lie algebra $\mathfrak{G}_{4}=\mathfrak{s l}(2, \mathbb{R})^{\oplus 3}$. Denoting by $X_{a}$ (raising operator), $Y_{a}$ (lowering operator), and $H_{a}:=\left[X_{a}, Y_{a}\right]$ the standard generators of the $a$-th $(a=1,2,3)$ copy of the Lie algebra $\mathfrak{s l}(2, \mathbb{R})$, the action of $\mathfrak{s l}(2, \mathbb{R}){ }^{\oplus 3}$ on a vector

\footnotetext{
${ }^{30}$ In 3 -centered BH physics, the $c_{i j}(i=1,2,3, j=1, \ldots, 8)$ spanning the $(\mathbf{3}, \mathbf{2}, \mathbf{2}, \mathbf{2})$ of $S L_{h}(3, \mathbb{R}) \times$ $S L(2, \mathbb{R})^{3}$ would usually be denoted as $Q_{a}^{M}$, with $a=1,2,3$ being the "horizontal" $S L_{h}(3, \mathbb{R})$-index, and $M=1, \ldots, 8$ denoting the $U$-duality $S L(2, \mathbb{R})^{3}$-index.
} 
$\left(c_{i 1}, \ldots, c_{i 8}\right) \in(\mathbf{2}, \mathbf{2}, \mathbf{2})$ can be realized through the identification $(i=1,2,3)^{31}$

$$
\begin{aligned}
& \left(c_{11}, \ldots, c_{18}\right)=\left(x_{000}, x_{001}, x_{010}, x_{011}, x_{100}, x_{101}, x_{110}, x_{111}\right) \\
& \left(c_{21}, \ldots, c_{28}\right)=\left(y_{000}, y_{001}, y_{010}, y_{011}, y_{100}, y_{101}, y_{110}, y_{111}\right) ; \\
& \left(c_{31}, \ldots, c_{38}\right)=\left(z_{000}, z_{001}, z_{010}, z_{011}, z_{100}, z_{101}, z_{110}, z_{111}\right)
\end{aligned}
$$

where the fundamental ( $\operatorname{spin} s=1 / 2$ ) irrep. $\mathbf{2}$ of $S L(2, \mathbb{R})$ is spanned by the indices $\mathbf{a}=0,1$. For example, the first copy of $\mathfrak{s l}(2, \mathbb{R})$ in $\mathfrak{s l}(2, \mathbb{R})^{\oplus 3}$ acts on the $\mathbf{x}_{\mathbf{a b c}}$ (equivalently denoting $x_{\mathbf{a b c}}$ or $y_{\mathbf{a b c}}$ or $\left.z_{\mathbf{a b c}}\right)$ as follows:

$$
X_{1} x_{\mathbf{a b c}}=\left\{\begin{array}{r}
0 \text { if } \mathbf{a}=0 ; \\
x_{0 \mathbf{b} \mathbf{c}} \text { if } \mathbf{a}=1 ;
\end{array} \quad Y_{1} x_{\mathbf{a b c}}=\left\{\begin{array}{r}
x_{1 \mathbf{b c}} \text { if } \mathbf{a}=0 ; \\
0 \text { if } \mathbf{a}=1 ;
\end{array} \quad H_{1} x_{\mathbf{a b c}}=\left\{\begin{array}{r}
x_{\mathbf{a b c}} \text { if } \mathbf{a}=0 ; \\
-x_{\mathbf{a b c}} \text { if } \mathbf{a}=1,
\end{array}\right.\right.\right.
$$

and similarly for the other two copies.

Then, one can compute the action of $\mathfrak{s l}(2, \mathbb{R})^{\oplus 3}$ on the Plücker coordinates (4.21), exploiting the fact that elements of $\mathfrak{s l}(2, \mathbb{R})^{\oplus 3}$ act as derivations on the $p_{i_{1} i_{2} i_{3}}$ 's themselves. For example, by using the identification (4.22), the action of $X_{1}$ of the first copy of $\mathfrak{s l}(2, \mathbb{R})$ in $\mathfrak{s l}(2, \mathbb{R})^{\oplus 3}$ on $p_{167}(4.21)$ reads

$$
X_{1} p_{167}=X_{1} \operatorname{det}\left(\begin{array}{lll}
x_{000} & y_{000} & z_{000} \\
x_{101} & y_{101} & z_{101} \\
x_{110} & y_{110} & z_{110}
\end{array}\right)=\operatorname{det}\left(\begin{array}{lll}
x_{000} & y_{000} & z_{000} \\
x_{001} & y_{001} & z_{001} \\
x_{110} & y_{110} & z_{110}
\end{array}\right)+\operatorname{det}\left(\begin{array}{lll}
x_{000} & y_{000} & z_{000} \\
x_{101} & y_{101} & z_{101} \\
x_{010} & y_{010} & z_{010}
\end{array}\right) ;
$$

therefore, by using the antisymmetry of the Plücker coordinates (4.21), one finds that $X_{1} p_{167}=p_{127}-p_{136}$. In this way, one can compute the action of each of the 9 generators $\left\{X_{1}, Y_{1}, H_{1}, X_{2}, Y_{2}, H_{2}, X_{3}, Y_{3}, H_{3}\right\}$ of $\mathfrak{s l}(2, \mathbb{R})^{\oplus 3}$ on the representation $\wedge^{3}(\mathbf{2}, \mathbf{2}, \mathbf{2})$ (realized in terms of Plücker coordinates (4.21); also cfr. (3.8)) of $S L(2, \mathbb{R})^{3}$. Such an action then extends to an action by derivations on polynomials in the $p_{i_{1} i_{2} i_{3}}$ 's themselves.

\subsubsection{The representation $V\left(a_{1}, a_{2}, a_{3}\right)$}

Let us now consider the realization of the representation $V\left(a_{1}, a_{2}, a_{3}\right)$ of $G_{4}=S L(2, \mathbb{R})^{3}$ on the space of homogeneous polynomials; here, we use the standard notation in which $V\left(a_{1}, a_{2}, a_{3}\right):=\left(\mathbf{a}_{1}+\mathbf{1}, \mathbf{a}_{2}+\mathbf{1}, \mathbf{a}_{3}+\mathbf{1}\right)$, and thus it has (real) dimension $\left(a_{1}+1\right)\left(a_{2}+1\right)\left(a_{3}+1\right)$ (namely, $\left(a_{1}, a_{2}, a_{3}\right)$ denote the weights of the vector space $V$ as $S L(2, \mathbb{R})^{3}$-representation).

The highest weight vector $v \in V\left(a_{1}, a_{2}, a_{3}\right)$ satisfies

$$
\left\{\begin{array}{l}
H_{i} v=a_{i} v ; \quad i=1,2,3 \\
X_{i} v=0
\end{array}\right.
$$

Thus, $V\left(a_{1}, a_{2}, a_{3}\right)$ can be realized as the vector space spanned by certain combinations of powers of lowering operators $X_{i}$ 's on its highest weight vector $v$ itself:

$$
V\left(a_{1}, a_{2}, a_{3}\right)=\left\langle Y_{1}^{k} Y_{2}^{l} Y_{3}^{m} v: 0 \leq k \leq a_{1}, 0 \leq l \leq a_{2}, 0 \leq m \leq a_{3}\right\rangle .
$$

\footnotetext{
${ }^{31}$ In physics literature, the basis $\left\{x_{\mathbf{a b c}}\right\}_{\mathbf{a}, \mathbf{b}, \mathbf{c}=0,1}$ is named qubit basis, because it naturally occurs in the quantum entanglement of three qubits in Quantum Information Theory. For relation to other symplectic frames in the stu model as well as recent developments related to the BH/qubit correspondence, see e.g. [49, $50,81-83]$ and [76-80], respectively.
} 
By virtue of (4.25), the vector $Y_{1}^{k} Y_{2}^{l} Y_{3}^{m} v \in V\left(a_{1}, a_{2}, a_{3}\right)$ is again an eigenvector of all three $H_{i}$ 's with weight $\left(a_{1}-2 k, a_{2}-2 l, a_{3}-2 m\right)$.

We are now going to exploit this general description in order to explicitly construct the $10\left(S L_{h}(3, \mathbb{R}) \times S L(2, \mathbb{R})^{3}\right)$-invariant homogeneous polynomials of degree 12 in the $(\mathbf{3}, \mathbf{2}, \mathbf{2}, \mathbf{2})$ considered in sections 4.1 and 4.2 , which constitute a complete basis for the corresponding 10-dimensional vector space resulting from table (2.45).

\subsubsection{The $(2,2,2)$ in $S_{1^{3}}((2,2,2))$}

Below (4.13), we observed that there is a (unique) irreducible tri-fundamental $S L(2, \mathbb{R})^{3}$ representation $V(1,1,1)=:(\mathbf{2}, \mathbf{2}, \mathbf{2})$ in $S_{1^{3}}((\mathbf{2}, \mathbf{2}, \mathbf{2}))=\wedge^{3}(\mathbf{2}, \mathbf{2}, \mathbf{2})=:(\mathbf{2}, \mathbf{2}, \mathbf{2})_{a}^{\otimes 3}$. In order to characterize it, we here determine its highest weight vector.

Besides $(\mathbf{2}, \mathbf{2}, \mathbf{2})$, also each of the other 3 irreducible summands of $S_{1^{3}}((\mathbf{2}, \mathbf{2}, \mathbf{2}))$ in the r.h.s. of (4.13) has a vector with weight $(1,1,1)$, therefore the weight space $S_{1^{3}}((\mathbf{2}, \mathbf{2}, \mathbf{2}))_{(1,1,1)}$ is four-dimensional:

$$
\begin{aligned}
S_{1^{3}}((\mathbf{2}, \mathbf{2}, \mathbf{2}))_{(1,1,1)}: & =\left\{v \in S_{1^{3}}((\mathbf{2}, \mathbf{2}, \mathbf{2})): H_{i} v=v, \quad i=1,2,3\right\} \\
& =\left\langle p_{145}, p_{136}, p_{235}, p_{127}\right\rangle,
\end{aligned}
$$

as one can check within the conventions adopted above. The unique (up to a scalar multiple) highest weight vector in this space is

$$
v:=p_{145}-p_{136}-p_{127}, \quad \text { so } \quad\langle v\rangle=\cap_{i=1}^{3} \operatorname{ker}\left(X_{i}\right) \cap S_{1^{3}}((\mathbf{2}, \mathbf{2}, \mathbf{2}))_{(1,1,1)} .
$$

Thus, an isomorphism between $(\mathbf{2}, \mathbf{2}, \mathbf{2}) \subset S_{1^{3}}((\mathbf{2}, \mathbf{2}, \mathbf{2}))$ and $(\mathbf{2}, \mathbf{2}, \mathbf{2})$ itself can be obtained, by setting

$$
x_{k l m}:=Y_{1}^{k} Y_{2}^{l} Y_{3}^{m} v, \quad k, l, m \in\{0,1\},
$$

where $v$ is defined in (4.28).

The usual expression of the $S L(2, \mathbb{R})^{3}$-invariant Cayley's hyperdeterminant $\mathcal{I}_{4}[54,55]$ in the tri-fundamental $(\mathbf{2}, \mathbf{2}, \mathbf{2})$ as a quartic homogeneous polynomial in the $x_{i j k}$ 's [56] (in qubit basis; cfr. footnote 30) produces a degree-4 polynomial in the Plücker coordinates $p_{i j k}(t)$ (4.21). As a polynomial in the $c_{i j}$ (cfr. e.g. the first line of (4.22)), such a polynomial is then $\left(S L_{h}(3, \mathbb{R}) \times S L(2, \mathbb{R})^{3}\right)$-invariant homogeneous of degree 12 in the $(\mathbf{3}, \mathbf{2}, \mathbf{2}, \mathbf{2})$; indeed, as expected, one can check that it coincides with the invariant $F_{0}(t)(4.11)$.

\subsubsection{The $(1,1,3)^{\oplus 3}$ in $S_{2^{3}}((2,2,2))$}

The $S L(2, \mathbb{R})^{3}$-representation $S_{2^{3}}((\mathbf{2}, \mathbf{2}, \mathbf{2}))$ is a sub-representation of $S^{2}\left(S_{1^{3}}((\mathbf{2}, \mathbf{2}, \mathbf{2}))\right)=$ : $\left((\mathbf{2}, \mathbf{2}, \mathbf{2})_{a}^{\otimes 3}\right)_{s}^{\otimes 2}$, which is the space of homogenous polynomials of degree 2 in the Plücker coordinates $p_{i j k}(t)$; in fact, by substituting the cubic polynomials (4.21) in the $c_{i j}$ for these $p_{i j k}$, one gets a vector space of degree-6 homogeneous polynomials in the $c_{i j}$ 's, which is nothing but $S_{2^{3}}((\mathbf{2}, \mathbf{2}, \mathbf{2}))$.

Using this fact, one can first determine the weight space

$$
\begin{aligned}
S^{2}\left(S_{1^{3}}((\mathbf{2}, \mathbf{2}, \mathbf{2}))\right)_{(0,0,2)}: & =\left\{v \in S^{2}\left(S_{1^{3}}((\mathbf{2}, \mathbf{2}, \mathbf{2}))\right): H_{i} v=0, \quad i=1,2, \quad H_{3} v=2 v\right\} \\
& =\left\langle p_{168} p_{137}, \ldots\right\rangle,
\end{aligned}
$$

which has dimension 52 . 
Next, by computing the images of the 52 basis elements under the raising operators $X_{i}, i=1,2,3$, one finds the highest weight vectors in $S^{2}\left(S_{1^{3}}((\mathbf{2}, \mathbf{2}, \mathbf{2}))\right)(4.30)$, which result to span a 5-dimensional sub-space of such a weight space. As they are rather complicated (and not particularly illuminating) homogeneous polynomials of degree 2 in the Plücker coordinates $p_{i j k}(t)$, we will refrain from reporting them here explicitly.

Then, by recalling (4.21), one can express $p_{i j k}$ in terms of the coordinates $c_{i j}$, thus obtaining a 3 -dimensional sub-space. Let $\left\{g_{1}, g_{2}, g_{3}\right\}$ be a basis of this sub-space; therefore, $(\mathbf{1}, \mathbf{1}, \mathbf{3})^{\oplus 3} \subset S_{2^{3}}((\mathbf{2}, \mathbf{2}, \mathbf{2}))$ (cfr. (4.18)) is spanned by $\left\{g_{i}, Y_{3}\left(g_{i}\right), Y_{3}^{2}\left(g_{i}\right)\right\}_{i=1,2,3}$. The $S L(2, \mathbb{R})^{3}$-representation $V(0,0,2)=:(\mathbf{1}, \mathbf{1}, \mathbf{3})$ has a unique invariant in $S^{2} V(0,0,2)=$ : $(\mathbf{1}, \mathbf{1}, \mathbf{3})_{s}^{\otimes 2}$, given by the Cartan-Killing metric in the adjoint $(\operatorname{spin} s=1)$ irrep. $\mathbf{3}$ of the third copy of $S L(2, \mathbb{R})$ in $S L(2, \mathbb{R})^{3}$ itself, and whose expression in terms of $\left\{g, Y_{3}(g), Y_{3}^{2}(g)\right\}$ is given by

$$
2 g Y_{3}^{2}(g)-\left(Y_{3}(g)\right)^{2}
$$

Thus, in $S^{2}\left(S_{2^{3}}((\mathbf{2}, \mathbf{2}, \mathbf{2}))\right) \subset S_{4^{3}}((\mathbf{2}, \mathbf{2}, \mathbf{2}))$, one gets $3\left(S L_{h}(3, \mathbb{R}) \times S L(2, \mathbb{R})^{3}\right)$ invariant homogeneous polynomials of degree 12 in the $(\mathbf{3}, \mathbf{2}, \mathbf{2}, \mathbf{2})$, which can be checked to be linearly independent as polynomials in the $c_{i j}$ 's.

By considering also the results of the same procedure repeated for $(\mathbf{3}, \mathbf{1}, \mathbf{1})^{\oplus 3} \subset$ $S_{2^{3}}((\mathbf{2}, \mathbf{2}, \mathbf{2}))$ as well as for $(\mathbf{1}, \mathbf{3}, \mathbf{1})^{\oplus 3} \subset S_{2^{3}}((\mathbf{2}, \mathbf{2}, \mathbf{2}))$ (cfr. (4.18)), one obtains a total of $3 S L(2, \mathbb{R})^{3}$-invariant homogeneous polynomials of degree 12 in $S^{2}\left(S_{2^{3}}((\mathbf{2}, \mathbf{2}, \mathbf{2}))\right) \subset$ $S_{4^{3}}((\mathbf{2}, \mathbf{2}, \mathbf{2}))$.

\subsection{4 stu Triality}

In order to determine the remaining relevant 6 invariants of degree 12, one can now use the action of the symmetric group $S_{3}$ on $R=(\mathbf{2}, \mathbf{2}, \mathbf{2})$ by permuting the tensor components, so $(12) \in S_{3}$ will map $x_{a b c}$ to $x_{b a c}$, etc. Consequently, $S_{3}$ will also act on the $c_{i j}$ 's, as well as on the Plücker coordinates $p_{i j k}$. As we will see below, in the context of $s t u$ black holes, the invariance under $S_{3}$ must be enforced, because it corresponds to the triality symmetry $[49,50]$ exhibited by such a model of $\mathcal{N}=2, D=4$ supergravity.

Using this action, the 3 invariants just found in section 4.3 .3 give rise to the required set of 9 invariants.

Including the invariant from section 4.1 (which, as mentioned above, matches the one obtained in section 4.3.2), one gets a total of 10 invariants of degree 12 in the $c_{i j}$ 's.

Thus, we constructed a basis $\left\{\mathbf{I}_{12, \alpha}\right\}_{\alpha=1, \ldots, 10}$ for the 10-dimensional vector space of $\left(S L_{h}(3, \mathbb{R}) \times S L(2, \mathbb{R})^{3}\right)$-invariant homogeneous polynomials of degree 12 in the $(\mathbf{3}, \mathbf{2}, \mathbf{2}, \mathbf{2})$ (resulting from table $(2.45))$.

As degree-12 homogeneous polynomials in the $c_{i j} \in(\mathbf{3}, \mathbf{2}, \mathbf{2}, \mathbf{2})$ of $S L_{h}(3, \mathbb{R}) \times$ $S L(2, \mathbb{R})^{3}$ (realized e.g. through the identification (4.22)), they have far too many terms, rendering their explicit expression cumbersome and not particularly illuminating. However, we observe that each of the invariants $\mathbf{I}_{12, \alpha}$ can be rewritten as

$$
\mathbf{I}_{12, \alpha}=\sum_{\beta=1}^{10} C_{\alpha \beta} M_{\beta}+\ldots,
$$


where $C_{\alpha \beta} \in \mathbb{Z}$, and $M_{\beta}(\beta=1, \ldots, 10)$ denotes the following set of monomials:

$$
\begin{array}{rll}
M_{1}:=c_{18}^{4} c_{23}^{2} c_{25} c_{26} c_{31}^{3} c_{32}, & M_{2}:=c_{18}^{4} c_{23}^{2} c_{25}^{2} c_{31}^{2} c_{32}^{2}, & M_{3}:=c_{18}^{4} c_{22} c_{24} c_{25}^{2} c_{31}^{3} c_{33}, \\
M_{4}:=c_{18}^{4} c_{22} c_{23} c_{26} c_{27} c_{31}^{4}, & M_{5}:=c_{18}^{4} c_{22} c_{23} c_{25} c_{27} c_{31}^{3} c_{32}, & M_{6}:=c_{18}^{4} c_{22}^{2} c_{27}^{2} c_{31}^{4}, \\
M_{7}:=c_{11}^{3} c_{16} c_{22}^{2} c_{27}^{2} c_{33} c_{38}^{3}, & M_{8}:=c_{11}^{3} c_{14} c_{22}^{2} c_{27}^{2} c_{35} c_{38}^{3}, & M_{9}:=c_{11}^{3} c_{14} c_{22} c_{23} c_{26} c_{27} c_{35} c_{38}^{3}, \\
M_{10}:=c_{11}^{3} c_{14} c_{23}^{2} c_{26}^{2} c_{35} c_{38}^{3}, &
\end{array}
$$

which completely characterize each $\mathbf{I}_{12, \alpha}$. Indeed, the dots in the right-hand side of (4.32) stand for many other linear combinations of monomials which are linearly independent on the $M_{\beta}$ 's, but which can be determined by the action of the whole group $S L_{h}(3, \mathbb{R}) \times$ $S L(2, \mathbb{R})^{3}$ itself, once the $C_{\alpha \beta}$ 's are specified. Of course, there are many sets of 10 monomials with the property (4.32). Given the set $M_{\beta}$ (4.33), each element $\mathbf{I}_{12, \alpha}$ of the 10-dimensional complete basis $\left\{\mathbf{I}_{12, \alpha}\right\}_{\alpha=1, \ldots, 10}$ constructed above can be written as a vector in $\mathbb{Z}^{10}$.

Let us make some examples.

The invariant $F_{0}$ (4.11), constructed in section 4.1 as well as in section 4.3 .2 , has coordinates $^{32}$

$$
F_{0} \equiv(0,0,0,-2,4,1,4,4,-8,4,4)
$$

On the other hand, the following three vectors correspond to the aforementioned basis $\left\{g_{1}, g_{2}, g_{3}\right\}$ the 3 -dimensional sub-space of invariants obtained from $(\mathbf{1}, \mathbf{1}, \mathbf{3})^{\oplus 3} \subset$ $S_{2^{3}}((\mathbf{2}, \mathbf{2}, \mathbf{2}))$ (cfr. section 4.3.3):

$$
\begin{aligned}
& g_{1} \equiv(-6,6,1,0,0,0,0,0,9,-9,-9) ; \\
& g_{2} \equiv(2,-2,-1,2,-4,-2,10,10,-15,5,5) ; \\
& g_{3} \equiv(2,-1,0,2,-2,-1,6,4,-8,4,4) .
\end{aligned}
$$

As mentioned above, the stu triality symmetry (implemented as the symmetric group $\left.S_{3}\right)$ permutes the subspaces $(\mathbf{1}, \mathbf{1}, \mathbf{3})^{\oplus 3},(\mathbf{1}, \mathbf{3}, \mathbf{1})^{\oplus 3}$ and $(\mathbf{3}, \mathbf{1}, \mathbf{1})^{\oplus 3}$; as a consequence, there is a 3 -dimensional sub-space of triality-invariant $\left(S L_{h}(3, \mathbb{R}) \times S L(2, \mathbb{R})^{3}\right)$-invariants in their direct sum, ${ }^{33}$ which is the space spanned by the vectors

$$
\begin{aligned}
& F_{1} \equiv(1,-1,1,1,-2,-1,3,3,-14,3,3) ; \\
& F_{2} \equiv(-5,5,-5,7,-14,-1,-7,-7,-10,-7,-7) ; \\
& F_{3} \equiv(4,-3,0,4,-6,-3,4,2,-22,8,8) .
\end{aligned}
$$

By adding the invariant $F_{0}$ (4.11) (or equivalently, through (4.32)-(4.33), (4.34)), which is also triality-invariant, out of $\left\{\mathbf{I}_{12, \alpha}\right\}_{\alpha=1, \ldots, 10}$ one gets a 4-dimensional basis $\left\{F_{0}, F_{1}, F_{2}, F_{3}\right\}$ for degree-12 homogeneous polynomials invariant under the action of

${ }^{32}$ What we actually write in $(4.34),(4.35)$ and $(4.36)$ are 11 integers $\left(a_{1}, \ldots, a_{11}\right)$ such that

$$
\sum_{\alpha=1}^{10} a_{\alpha} \mathbf{I}_{12, \alpha}+a_{11} F_{0}=0
$$

${ }^{33}$ Its complement is the direct sum of three 2-dimensional (irreducible) $S_{3}$-representations. 
$S_{3} \times S L_{h}(3, \mathbb{R}) \times S L(2, \mathbb{R})^{3}$ on $(\mathbf{3}, \mathbf{2}, \mathbf{2}, \mathbf{2})$. Thus, as anticipated in section 4,4 is the (real) dimension of the vector space of degree-12 $\left(S L_{h}(3, \mathbb{R}) \times S L(2, \mathbb{R})^{3}\right)$-invariant polynomials relevant for the 3 -centered $\mathrm{BHs}$ in the $\mathcal{N}^{\prime}=2, D=4$ stu model.

\section{Acknowledgments}

We would like to thank Sergio Ferrara for useful discussions and correspondence.

A. M. would also like to thank Giuseppe Torri for interesting discussions on the Hilbert series and plethystic logarithm.

Open Access. This article is distributed under the terms of the Creative Commons Attribution License which permits any use, distribution and reproduction in any medium, provided the original author(s) and source are credited.

\section{References}

[1] S. Ferrara, R. Kallosh and A. Strominger, $N=2$ extremal black holes, Phys. Rev. D 52 (1995) 5412 [hep-th/9508072] [INSPIRE].

[2] A. Strominger, Macroscopic entropy of $N=2$ extremal black holes, Phys. Lett. B 383 (1996) 39 [hep-th/9602111] [INSPIRE].

[3] S. Ferrara and R. Kallosh, Supersymmetry and attractors, Phys. Rev. D 54 (1996) 1514 [hep-th/9602136] [INSPIRE].

[4] S. Ferrara and R. Kallosh, Universality of supersymmetric attractors, Phys. Rev. D 54 (1996) 1525 [hep-th/9603090] [INSPIRE].

[5] S. Ferrara, G.W. Gibbons and R. Kallosh, Black holes and critical points in moduli space, Nucl. Phys. B 500 (1997) 75 [hep-th/9702103] [INSPIRE].

[6] L. Andrianopoli, R. D'Auria, S. Ferrara and M. Trigiante, Extremal black holes in supergravity, Lect. Notes Phys. 737 (2008) 661 [hep-th/0611345] [INSPIRE].

[7] S. Bellucci, S. Ferrara, R. Kallosh and A. Marrani, Extremal Black Hole and Flux Vacua Attractors, Lect. Notes Phys. 755 (2008) 115 [arXiv:0711.4547] [INSPIRE].

[8] S. Ferrara, K. Hayakawa and A. Marrani, Lectures on Attractors and Black Holes, Fortsch. Phys. 56 (2008) 993 [arXiv:0805.2498] [InSPIRE].

[9] G. Dall'Agata, Black holes in supergravity: flow equations and duality, arXiv:1106.2611 [INSPIRE].

[10] S. Ferrara, A. Marrani, J.F. Morales and H. Samtleben, Intersecting Attractors, Phys. Rev. D 79 (2009) 065031 [arXiv:0812.0050] [INSPIRE].

[11] G.W. Moore, Arithmetic and attractors, hep-th/9807087 [INSPIRE].

[12] R. Kallosh, A.D. Linde and M. Shmakova, Supersymmetric multiple basin attractors, JHEP 11 (1999) 010 [hep-th/9910021] [INSPIRE].

[13] A. Giryavets, New attractors and area codes, JHEP 03 (2006) 020 [hep-th/0511215] [INSPIRE].

[14] S. Ferrara and A. Marrani, On the Moduli Space of non-BPS Attractors for $N=2$ Symmetric Manifolds, Phys. Lett. B 652 (2007) 111 [arXiv:0706.1667] [InSPIRE]. 
[15] E. Cremmer and B. Julia, The $N=8$ Supergravity Theory. 1. The Lagrangian, Phys. Lett. B 80 (1978) 48 [INSPIRE].

[16] E. Cremmer and B. Julia, The SO(8) Supergravity, Nucl. Phys. B 159 (1979) 141 [inSPIRE].

[17] C. Hull and P. Townsend, Unity of superstring dualities, Nucl. Phys. B 438 (1995) 109 [hep-th/9410167] [INSPIRE].

[18] V.G. Kac, Some Remarks on Nilpotent Orbits, J. Algebra 64 (1980) 190.

[19] S. Ferrara and M. Günaydin, Orbits of exceptional groups, duality and BPS states in string theory, Int. J. Mod. Phys. A 13 (1998) 2075 [hep-th/9708025] [INSPIRE].

[20] S. Bellucci, S. Ferrara, M. Günaydin and A. Marrani, Charge orbits of symmetric special geometries and attractors, Int. J. Mod. Phys. A 21 (2006) 5043 [hep-th/0606209] [INSPIRE].

[21] A. Ceresole, S. Ferrara and A. Marrani, Small $N=2$ Extremal Black Holes in Special Geometry, Phys. Lett. B 693 (2010) 366 [arXiv:1006.2007] [INSPIRE].

[22] L. Borsten, M. Duff, S. Ferrara, A. Marrani and W. Rubens, Small Orbits, Phys. Rev. D 85 (2012) 086002 [arXiv:1108.0424] [InSPIRE].

[23] A. Marrani, Charge Orbits and Moduli Spaces of Black Hole Attractors, Lect. Notes Math. 2027 (2011) 155 [arXiv: 1012.3559] [INSPIRE].

[24] J. Luciani, Coupling of $O(2)$ Supergravity with Several Vector Multiplets, Nucl. Phys. B 132 (1978) 325 [nSPIRE].

[25] S. Ferrara, A. Gnecchi and A. Marrani, D =4 Attractors, Effective Horizon Radius and Fake Supergravity, Phys. Rev. D 78 (2008) 065003 [arXiv:0806.3196] [INSPIRE].

[26] L. Castellani, A. Ceresole, S. Ferrara, R. D'Auria, P. Fré and E. Maina, The complete $N=3$ matter coupled supergravity, Nucl. Phys. B 268 (1986) 317 [INSPIRE].

[27] R.B. Brown, Groups of type $E_{7}$, J. Reine Angew. Math. 236 (1969) 79.

[28] K. Meyberg, Eine Theorie der Freudenthalschen Triplesysteme. I, II, Nederl. Akad. Wetensch. Proc. Ser. A 71 (1968) 162.

[29] R.S. Garibaldi, Groups of type $E_{7}$ over arbitrary fields, math/9811056.

[30] L. Borsten, D. Dahanayake, M. Duff and W. Rubens, Black holes admitting a Freudenthal dual, Phys. Rev. D 80 (2009) 026003 [arXiv:0903.5517] [inSPIRE].

[31] S. Ferrara and R. Kallosh, Creation of Matter in the Universe and Groups of Type $E_{7}$, JHEP 12 (2011) 096 [arXiv: 1110.4048] [INSPIRE].

[32] S. Ferrara, R. Kallosh and A. Marrani, Degeneration of Groups of Type $E_{7}$ and Minimal Coupling in Supergravity, JHEP 06 (2012) 074 [arXiv: 1202.1290] [INSPIRE].

[33] S. Ferrara and A. Marrani, Black Holes and Groups of Type E ${ }_{7}$, Pramana 78 (2012) 893 [arXiv:1112.2664] [INSPIRE].

[34] A. Marrani, E. Orazi and F. Riccioni, Exceptional Reductions, J. Phys. A 44 (2011) 155207 [arXiv: 1012.5797] [INSPIRE].

[35] L. Andrianopoli, R. D'Auria, S. Ferrara, A. Marrani and M. Trigiante, Two-Centered Magical Charge Orbits, JHEP 04 (2011) 041 [arXiv:1101.3496] [INSPIRE].

[36] A. Ceresole, S. Ferrara, A. Marrani and A. Yeranyan, Small Black Hole Constituents and Horizontal Symmetry, JHEP 06 (2011) 078 [arXiv: 1104.4652] [INSPIRE]. 
[37] S. Ferrara, A. Marrani and A. Yeranyan, On Invariant Structures of Black Hole Charges, JHEP 02 (2012) 071 [arXiv:1110.4004] [INSPIRE].

[38] F. Denef, Supergravity flows and D-brane stability, JHEP 08 (2000) 050 [hep-th/0005049] [INSPIRE].

[39] B. Bates and F. Denef, Exact solutions for supersymmetric stationary black hole composites, JHEP 11 (2011) 127 [hep-th/0304094] [INSPIRE].

[40] D. Gaiotto, W. Li and M. Padi, Non-Supersymmetric Attractor Flow in Symmetric Spaces, JHEP 12 (2007) 093 [arXiv:0710.1638] [INSPIRE].

[41] E.G. Gimon, F. Larsen and J. Simon, Constituent Model of Extremal non-BPS Black Holes, JHEP 07 (2009) 052 [arXiv:0903.0719] [INSPIRE].

[42] P. Fré and A.S. Sorin, Extremal Multicenter Black Holes: Nilpotent Orbits and Tits Satake Universality Classes, JHEP 01 (2013) 003 [arXiv:1205.1233] [INSPIRE].

[43] K. Goldstein and S. Katmadas, Almost BPS black holes, JHEP 05 (2009) 058 [arXiv: 0812.4183] [INSPIRE].

[44] I. Bena, S. Giusto, C. Ruef and N.P. Warner, Multi-Center non-BPS Black Holes: the Solution, JHEP 11 (2009) 032 [arXiv:0908.2121] [INSPIRE].

[45] G. Bossard and C. Ruef, Interacting non-BPS black holes, Gen. Rel. Grav. 44 (2012) 21 [arXiv:1106.5806] [INSPIRE].

[46] G. Bossard, Octonionic black holes, JHEP 05 (2012) 113 [arXiv: 1203.0530] [INSPIRE].

[47] A. Yeranyan, Multi-Centered Black Hole Flows, JHEP 08 (2012) 158 [arXiv:1205.5618] [INSPIRE].

[48] S. Ferrara, A. Marrani, E. Orazi, R. Stora and A. Yeranyan, Two-Center Black Holes Duality-Invariants for STU Model and its lower-rank Descendants, J. Math. Phys. 52 (2011) 062302 [arXiv:1011.5864] [INSPIRE].

[49] M. Duff, J.T. Liu and J. Rahmfeld, Four-dimensional string-string-string triality, Nucl. Phys. B 459 (1996) 125 [hep-th/9508094] [InSPIRE].

[50] K. Behrndt, R. Kallosh, J. Rahmfeld, M. Shmakova and W.K. Wong, STU black holes and string triality, Phys. Rev. D 54 (1996) 6293 [hep-th/9608059] [INSPIRE].

[51] P. Levay, Two-Center Black Holes, Qubits and Elliptic Curves, Phys. Rev. D 84 (2011) 025023 [arXiv: 1104.0144] [inSPIRE].

[52] C. Procesi, Lie Groups : An approach through Invariants and Representations, Springer Verlag (2005).

[53] W. Fulton and J. Harris, Representation Theory, Springer Verlag (1991).

[54] A. Cayley, On the theory of linear transformations, Camb. Math. J. 4 (1845) 193.

[55] P. Gibbs, Diophantine Quadruples and Cayley's Hyperdeterminant, math/0107203.

[56] M. Duff, String triality, black hole entropy and Cayley's hyperdeterminant, Phys. Rev. D 76 (2007) 025017 [hep-th/0601134] [INSPIRE].

[57] P. Gibbs, Elliptic Curves and Hyperdeterminants in Quantum Gravity, arXiv:1010.4219 [INSPIRE]. 
[58] I. Bena, G. Dall'Agata, S. Giusto, C. Ruef and N.P. Warner, Non-BPS Black Rings and Black Holes in Taub-NUT, JHEP 06 (2009) 015 [arXiv:0902.4526] [INSPIRE].

[59] G. Dall'Agata, S. Giusto and C. Ruef, U-duality and non-BPS solutions, JHEP 02 (2011) 074 [arXiv: 1012.4803] [INSPIRE].

[60] S. Ferrara, A. Marrani and E. Orazi, Split Attractor Flow in $N=2$ Minimally Coupled Supergravity, Nucl. Phys. B 846 (2011) 512 [arXiv: 1010.2280] [InSPIRE].

[61] A. Marrani, C.-X. Qiu, S.-Y.D. Shih, A. Tagliaferro and B. Zumino, Freudenthal Gauge Theory, arXiv: 1208.0013 [INSPIRE].

[62] M.K. Gaillard and B. Zumino, Duality Rotations for Interacting Fields, Nucl. Phys. B 193 (1981) 221 [inSPIRE].

[63] E.B. Dynkin, The Maximal Subgroups of the Classical Groups, American Mathematical Society Translations Series 26 (1957) 245.

[64] M. Lorente and B. Gruber, Classification of semisimple subalgebras of simple lie algebras, J. Math. Phys. 13 (1972) 1639 [INSPIRE].

[65] M. Günaydin, G. Sierra and P. Townsend, Exceptional Supergravity Theories and the MAGIC Square, Phys. Lett. B 133 (1983) 72 [INSPIRE].

[66] M. Günaydin, G. Sierra and P. Townsend, The Geometry of $N=2$ Maxwell-Einstein Supergravity and Jordan Algebras, Nucl. Phys. B 242 (1984) 244 [INSPIRE].

[67] M. Günaydin, G. Sierra and P. Townsend, Gauging the D $=5$ Maxwell-Einstein Supergravity Theories: More on Jordan Algebras, Nucl. Phys. B 253 (1985) 573 [InSPIRE].

[68] L. Andrianopoli, R. D'Auria and S. Ferrara, U invariants, black hole entropy and fixed scalars, Phys. Lett. B 403 (1997) 12 [hep-th/9703156] [INSPIRE].

[69] D. Roest and H. Samtleben, Twin Supergravities, Class. Quant. Grav. 26 (2009) 155001 [arXiv:0904.1344] [INSPIRE].

[70] M. Günaydin, Generalized conformal and superconformal group actions and Jordan algebras, Mod. Phys. Lett. A 8 (1993) 1407 [hep-th/9301050] [INSPIRE].

[71] M. Günaydin, K. Koepsell and H. Nicolai, Conformal and quasiconformal realizations of exceptional Lie groups, Commun. Math. Phys. 221 (2001) 57 [hep-th/0008063] [INSPIRE].

[72] M. Günaydin and O. Pavlyk, Spectrum Generating Conformal and Quasiconformal U-duality Groups, Supergravity and Spherical Vectors, JHEP 04 (2010) 070 [arXiv:0901.1646] [INSPIRE].

[73] B. de Wit and H. Nicolai, $N=8$ Supergravity, Nucl. Phys. B 208 (1982) 323 [InSPIRE].

[74] P. Breitenlohner, D. Maison and G.W. Gibbons, Four-Dimensional Black Holes from Kaluza-Klein Theories, Commun. Math. Phys. 120 (1988) 295 [inSPIRE].

[75] P. Jordan, J. von Neumann and E. P. Wigner, On an algebraic generalization of the quantum mechanical formalism, Annals Math. 35 (1934) 29.

[76] L. Borsten, D. Dahanayake, M. Duff, W. Rubens and H. Ebrahim, Freudenthal triple classification of three-qubit entanglement, Phys. Rev. A 80 (2009) 032326 [arXiv: 0812.3322] [INSPIRE].

[77] B.L. Cerchiai and B. van Geemen, From qubits to E $E_{7}$, J. Math. Phys. 51 (2010) 122203 [arXiv: 1003.4255] [INSPIRE]. 
[78] L. Borsten, D. Dahanayake, M. Duff, A. Marrani and W. Rubens, Four-qubit entanglement from string theory, Phys. Rev. Lett. 105 (2010) 100507 [arXiv: 1005.4915] [INSPIRE].

[79] L. Borsten, M. Duff, A. Marrani and W. Rubens, On the Black-Hole/Qubit Correspondence, Eur. Phys. J. Plus 126 (2011) 37 [arXiv:1101.3559] [INSPIRE].

[80] L. Borsten, M. Duff and P. Levay, The black-hole/qubit correspondence: an up-to-date review, Class. Quant. Grav. 29 (2012) 224008 [arXiv:1206.3166] [INSPIRE].

[81] R. Kallosh and A.D. Linde, Strings, black holes and quantum information, Phys. Rev. D 73 (2006) 104033 [hep-th/0602061] [INSPIRE].

[82] S. Bellucci, A. Marrani, E. Orazi and A. Shcherbakov, Attractors with Vanishing Central Charge, Phys. Lett. B 655 (2007) 185 [arXiv:0707.2730] [INSPIRE].

[83] S. Bellucci, S. Ferrara, A. Marrani and A. Yeranyan, $D=4$ Black Hole Attractors in $N=2$ Supergravity with Fayet-Iliopoulos Terms, Phys. Rev. D 77 (2008) 085027 [arXiv: 0802.0141] [INSPIRE]. 